\title{
Cosmique cosmétique. Pour une cosmologie de la parure
}

Cosmical Cosmetics: For a Cosmology of Adornment

\section{Bertrand Prévost}

\section{(2) OpenEdition}

1 Journals

Édition électronique

URL : http://journals.openedition.org/imagesrevues/2181

DOI : 10.4000/imagesrevues.2181

ISSN : 1778-3801

\section{Éditeur :}

Centre d'Histoire et Théorie des Arts, Groupe d'Anthropologie Historique de l'Occident Médiéval, Laboratoire d'Anthropologie Sociale, UMR 8210 Anthropologie et Histoire des Mondes Antiques

\section{Référence électronique}

Bertrand Prévost, « Cosmique cosmétique. Pour une cosmologie de la parure », Images Re-vues [En ligne], 10 | 2012, mis en ligne le, consulté le 04 mars 2021. URL : http://journals.openedition.org/ imagesrevues/2181; DOI : https://doi.org/10.4000/imagesrevues.2181

Ce document a été généré automatiquement le 4 mars 2021.

Images Re-vues est mise à disposition selon les termes de la Licence Creative Commons Attribution Pas d'Utilisation Commerciale 4.0 International. 


\section{Cosmique cosmétique. Pour une cosmologie de la parure}

Cosmical Cosmetics: For a Cosmology of Adornment

\section{Bertrand Prévost}

Fig. 1 

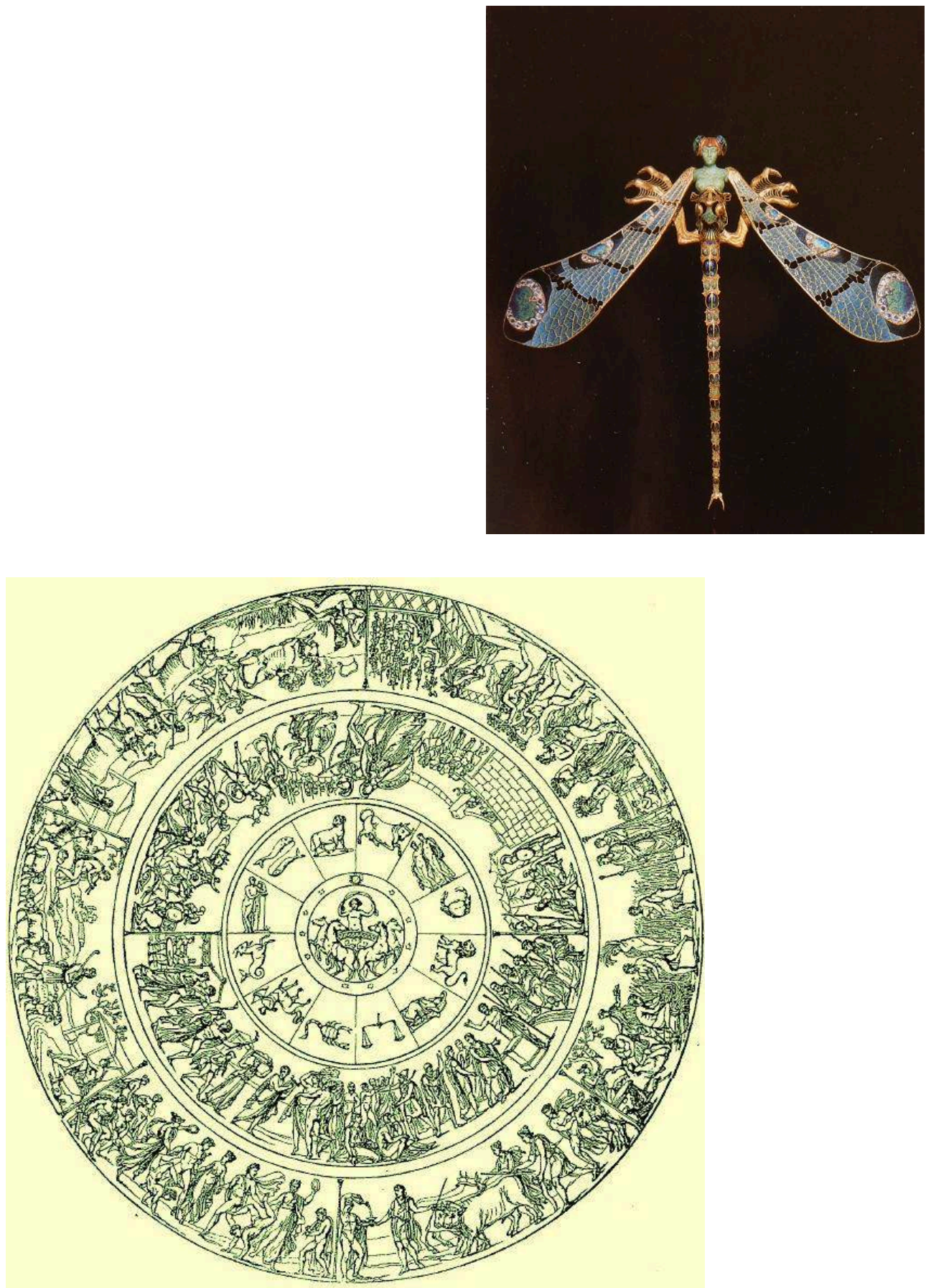

Le bouclier d'Achille, reconstitution française du XVIII' siècle, gravure

1 À quelles conditions la parure peut-elle faire l'objet d'une cosmologie ? En ce qu'il y a du cosmique dans la cosmétique, du mondain dans la parure. Les Grecs Anciens avaient développé tout un appareil sémantique et conceptuel pour recouvrir une telle articulation. Kosmos, en effet, signifie en grec aussi bien le monde, ou plutôt l'ordre du monde, que la parure, l'ornement corporel : ainsi, quand Héra se pare pour séduire Zeus, dans l'Illiade : " ayant ainsi orné son corps de toute sa parure (kosmon), elle quitta sa chambre $»^{1}$. Mais kosmos peut également renvoyer à l'équipement guerrier (le soldat est paré [kosmèthéis] de ses armes et armure, fig. 1), ou encore à l'apprêtage du mort, au silence comme ornement des femmes, etc. Bref, dans tous les cas de figure, il s'agit de 
penser l'ornement comme un plus qui n'est pas superflu mais harmonieusement complémentaire. "L'ornement, lorsqu'il est désigné par kosmos, renvoie à un artefact qui fait son apparition pour compléter le monde selon la convenance, un artefact (mais aussi bien éventuellement une attitude) qui à la fois, donc, "complète" et "convient" " 2 La beauté de la parure ne tient pas à une qualité intrinsèque mais à sa valeur d'ajustement, d'ajointement physique comme moral : une «belle ordonnance ».

Fig. 2

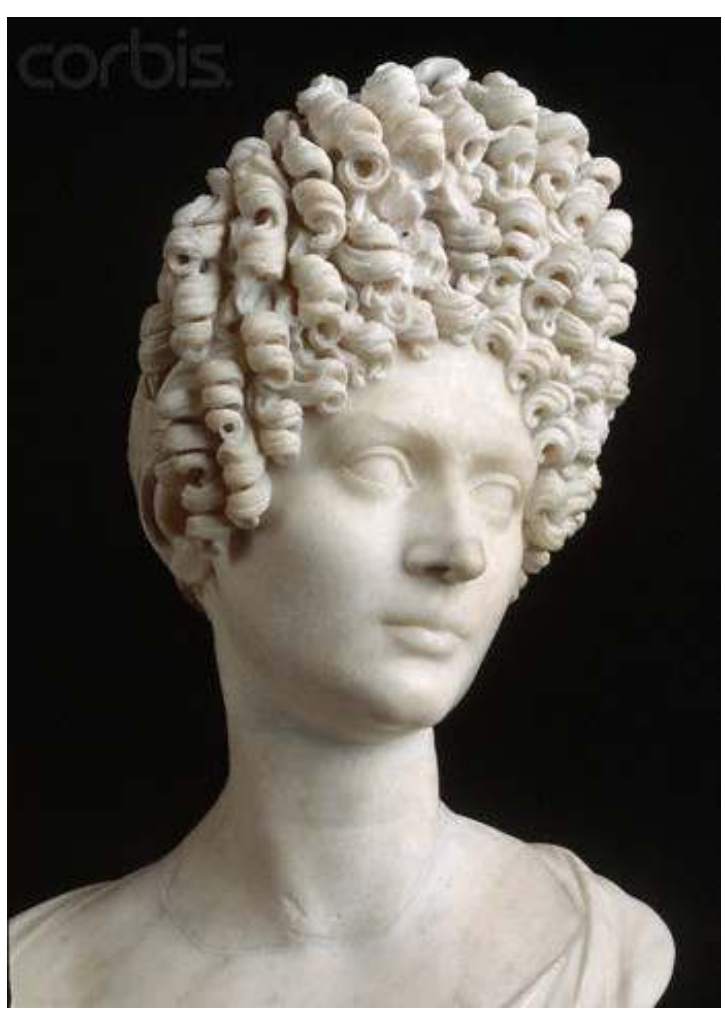

Buste Fonseca, IIe siècle ap. J.-C., marbre, Rome, Musées capitolins

2 Il n'y aura pas à s'étonner que les premiers Chrétiens aient vomi leur haine du monde et leur condamnation de la parure dans un même mouvement (fig. 2). À commencer, bien évidemment par Tertullien :

La toilette féminine présente un double aspect: la parure (cultum) et les soins de beauté (ornatum). Nous appelons "parure" ce qu'on nomme les atours des femmes (mundum muliebrem), "soins de beauté" ce qu'il faudrait appeler leur souillure (immundum muliebrum). La première consiste dans l'or, l'argent, les pierreries, le vêtement; les seconds dans le soin de la chevelure, de la peau et des parties du corps qui attirent les regards. Nous inculpons l'une d'orgueil, les autres de luxure ${ }^{3}$.

La position chrétienne ne recoupe plus ici l'opposition grecque du cosmos et du chaos, de l'ordre et du désordre, mais dénonce une pourriture intra-mondaine, par laquelle la parure ne fait plus monde mais devient, à la lettre, immondice. L'efficacité dialectique de cette critique de la parure consiste ainsi à ne plus opposer extérieurement l'ordonnancement cosmique et sa dislocation, mais à poser que le monde est gagné, de l'intérieur, par un travail du négatif qui le rend immonde : "la cosmétique est une cosmologie déchue $»^{4}$. Le raisonnement fonctionne de fait sur une inversion 
typiquement chrétienne; ainsi quand le même Tertullien, un peu plus loin dans son traité, condamne la parure au nom d'une plus authentique parure :

Grand blasphème, si l'on dit de quelqu'une: "Depuis qu'elle s'est faite chrétienne, son allure est plus pauvre"! Craindras-tu de paraître plus pauvre (pauperior), quand tu es devenue plus riche (locupletior), ou plus négligée (sordidior) quand tu es plus parée (mundior) ? L'allure des chrétiens doit-elle se régler sur le bon plaisir des païens ou sur celui de Dieu ?5

Il faut comprendre que si la parure des femmes revient à une souillure, c'est parce que la véritable parure tient, elle, dans le dépouillement: faire de sa nudité son plus pur vêtement, à l'instar de la nudité adamique et christique : « suivre nu le Christ nu (nudus nudum Christum sequi) $»^{6}$.

\section{L'ordre de l'ornement corporel}

5 Toujours est-il que la mondanité de l'ornement corporel en passe fondamentalement par une question d'ordre. Du cosmique à la cosmétique, c'est un même ordre qui s'étend à toutes les échelles, des sphères célestes jusqu'aux plus petites perles de collier, des trajectoires astrales jusqu'à la régularité des stries laissées par le passage du peigne dans les cheveux. On tient là, sans doute, ce qui des Grecs jusqu'à nous travaille en profondeur toute idée de cosmétique. C'est que toutes les théories de l'ornement corporel, voire de l'ornement tout court, s'inscrivent dans cette conception de l'ordre : des Anciens à Leon Battista Alberti et les Renaissants (en incluant les médiévaux), des théoriciens du XIX ${ }^{e}$ siècle (de Gottfried Semper à Charles Blanc) aux modernes historiens et théoriciens de l'art (de Ernst Gombrich, Jacques Soulillou à Jean-Claude Bonne, pour ne citer qu'eux), il s'agit toujours de penser l'ornement par-delà sa substantialisation dans un "vocabulaire ", et en le voyant davantage comme le travail d'un ornemental ${ }^{7}$ tenant à un ordre structural.

Si l'ornement sert à mettre de l'ordre, écrit Jacques Soulillou, c'est au sens où il permet à l'ordre d'apparaitre, comme on dit d'une chose qu'elle apparait dans la lumière. [...] L'apparition d'un ordre au sein d'un milieu constitué d'une myriade d'ornements est comparable au phénomène de la cristallisation où, à partir d'un germe, une structure se propage de proche en proche à tout un champ. La conjonction soudaine de l'ornement et de l'ordre dans un milieu donné produit un monde - kosmos. [...] Il y a kosmos lorsque l'ornement permet au "bon ordre" de se manifester. Est au contraire "acosmique" et "laid" ce qui dérange et trouble le bon ordre 8 .

6 Au-delà de l'ornement, c'est à l'art lui-même que s'attacherait cette question de l'ordre, au regard notamment d'un très ancien fonds indo-européen. Émile Benvéniste a ainsi mis au jour l'importance sémantique d'un concept indo-européen d'ordre :

C'est l'“Ordre" qui règle aussi bien l'ordonnance de l'univers, le mouvement des astres, la périodicité des saisons et des années que les rapports des hommes et des dieux, enfin des hommes entre eux. Rien de ce qui touche à l'homme, au monde, n'échappe à l'empire de l'“Ordre". C'est donc le fondement tant religieux que moral de toute société ; sans ce principe, tout retournerait au chaos ${ }^{9}$.

7 Or, c'est cette racine qui, parmi de très nombreux dérivés, a donné le grec ararískô («ajuster, adapter, harmoniser») aussi bien que le latin ars, artis (« disposition naturelle, qualification, talent »), artus (« articulation »), ritus (« ordonnance, rite ») ou encore le grec arthron («articulation, membre »). «Partout, commente Benvéniste, la 
même notion est encore sensible : l'ordonnance, l'ordre, l'adaptation étroite entre les

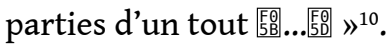

Fig. 3

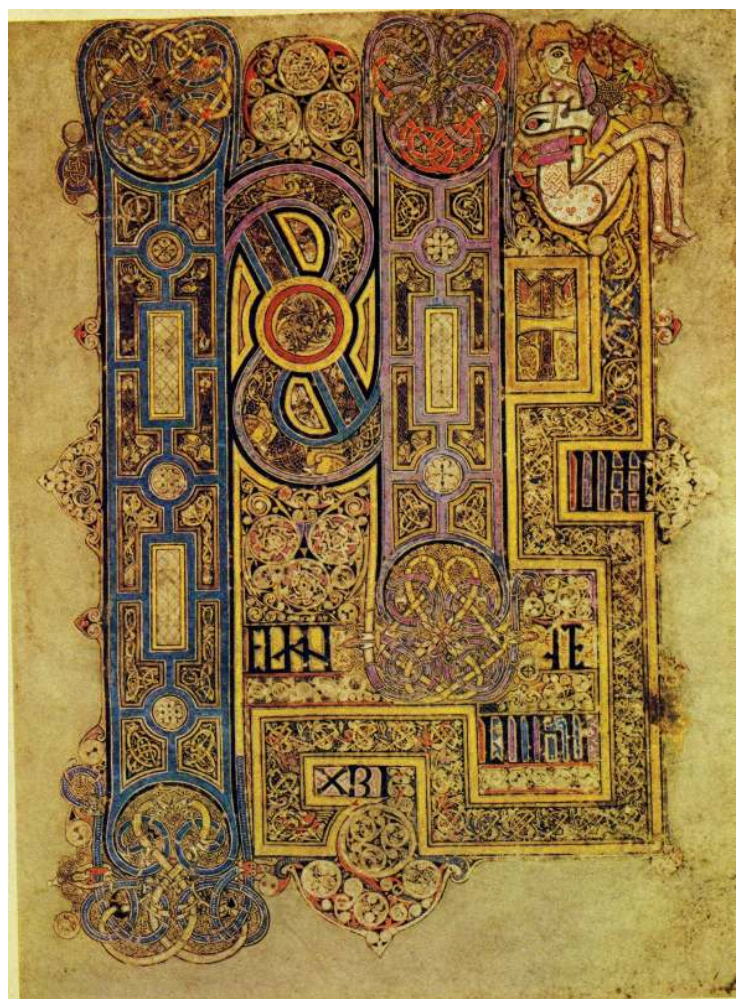

Livre de Kells, frontispice de l'évangile selon Saint Marc, IXe siècle, Dublin, Trinity College Library, Ms 58, $f^{\circ} 30 r$

8 L'ornemental médiéval chrétien (fig. 3) s'inscrit parfaitement dans cette esthétique de l'ordre universel, mais en tant qu'il

appelle moins une interprétation qui le fonderait qu'il ne fonde lui-même une pensée de l'ordre ou même de l'organisation [...]. C'est que cette pensée de l'ordre est naturellement indissociable dans l'herméneutique chrétienne de l'idée d'un principe créateur, et on peut l'interpréter comme une façon de définir les rapports "harmonieux" entre le créateur divin, ses créatures et tous les niveaux de la création. En sorte que l'esthétique ornementale médiévale est animée par la double exigence contradictoire ou du moins paradoxale d'un ordre à la fois mondain et divin, fini et infini (elle est travaillée par la similarité et la multiplication d'un 'comme à l'infini')"11. 
Fig. 4

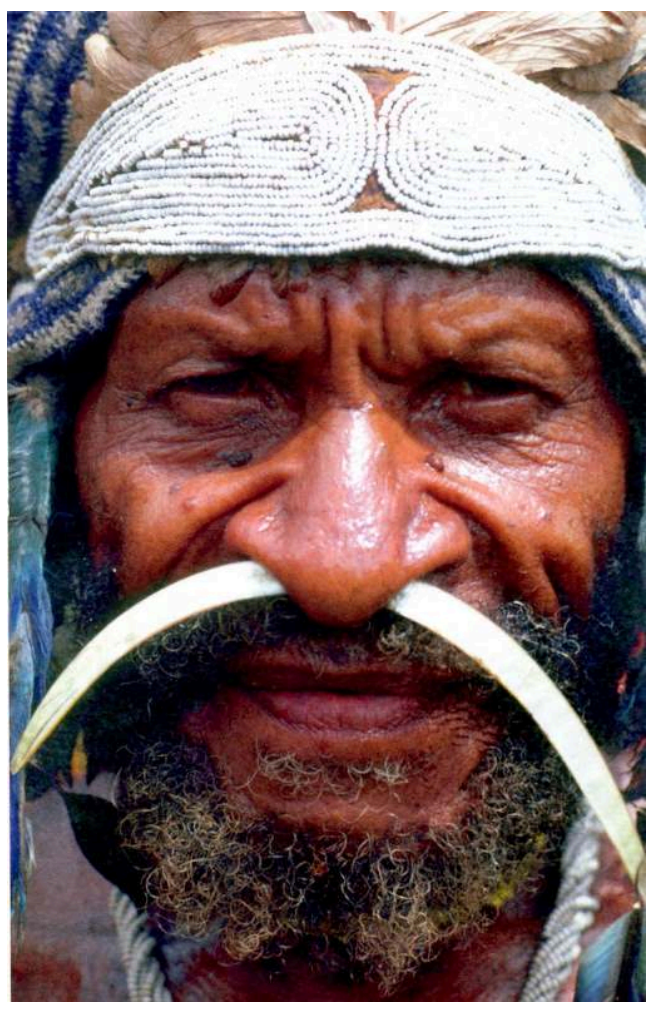

Ornement nasal, ivoire, Nouvelle-Guinée

9 On sait d'ailleurs de quel concept autant que de quel ressort esthétique le Moyen Age chrétien aura usé pour dire l'abstraction de ces rapports de proportion entre régions discontinues ou opposées : musica. Dans une inspiration que les médiévaux trouvaient chez saint Augustin autant que chez Boèce, la musica nommait un principe d'harmonie universelle s'articulant en mesure, nombre, rythme ${ }^{12}$. L'ornemental devient alors ce

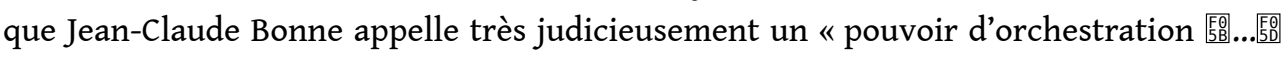
capable de fonctionner comme une sorte de transcodeur abstrait $»^{13}$. 
Fig. 5

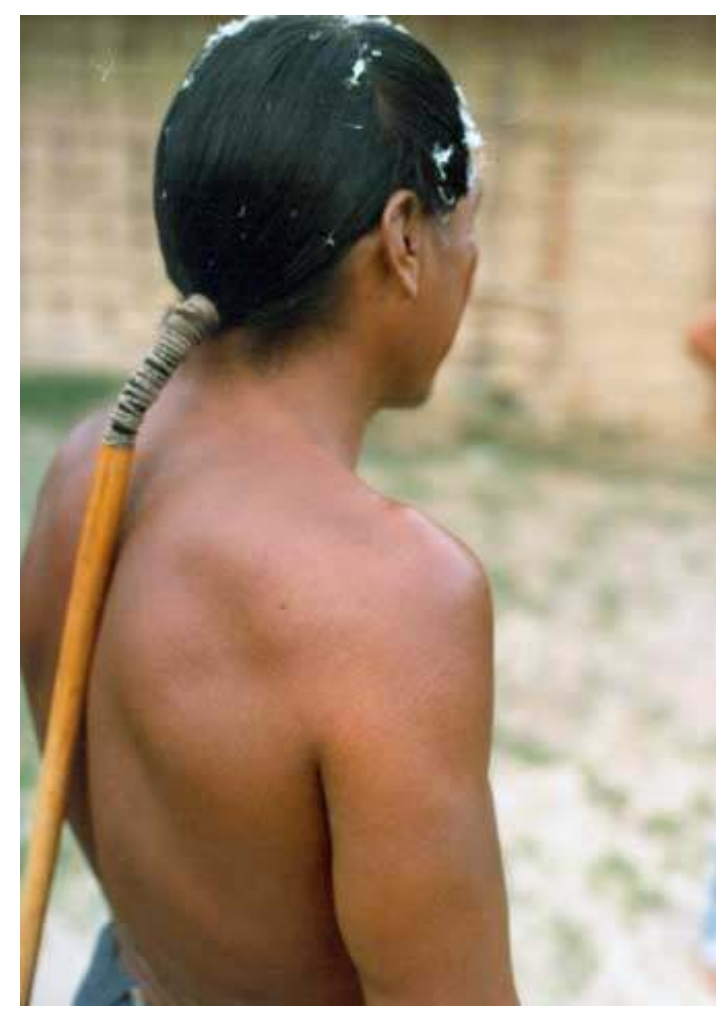

Indien Waiwai, Amazonie brésilienne

Gottfried Semper, au milieu du XIX ${ }^{e}$ siècle, est sans doute celui qui a poussé le plus loin « une pensée de l'ornement entendu selon cette dimension cosmique ${ }^{14}$, au titre de ce qu'il aura lui-même nommé une Kunstphysik, une "physique de l'art ${ }^{15}$. Ce que cherchait Semper était bien de l'ordre d'une « légalité naturelle ( Naturgesetzlichkeit), signifiant par là que les mêmes principes naturels doivent s'appliquer au fonctionnement du plus grand comme du plus petit, que les mêmes lois président aux formes naturelles comme aux formes artificielles. «Quand l'homme orne un objet, il ne fait qu'accentuer plus nettement de manière plus ou moins consciente une légalité naturelle déjà présente dans l'objet qu'il décore ${ }^{16}$. Mais surtout, on doit à Semper d'avoir articulé cette cosmologie de l'ornement non plus à l'ornement en général, mais plus précisément à l'ornement corporel, fournissant ainsi l'ébauche d'une vaste morphologie de la parure, en tant que cette morphologie n'aurait plus rien à voir avec une stricte typologie des formes et des matières mais avec les lignes de partage de trois grands principes physiques. Le premier ensemble concerne pour Semper «l'ornement pendant » (der Behang) : ornements de nez et d'oreille et autres breloques; ou encore le soin apporté à la barbe ou à la chevelure ou encore une draperie tombant en plis droits et réguliers (fig. 4,5). "Symétrique en lui-même, l'ornement pendant est lié de préférence à cette propriété formelle des phénomènes sensibles que nous appelons symétrie $»^{17}$. 
Fig. 6

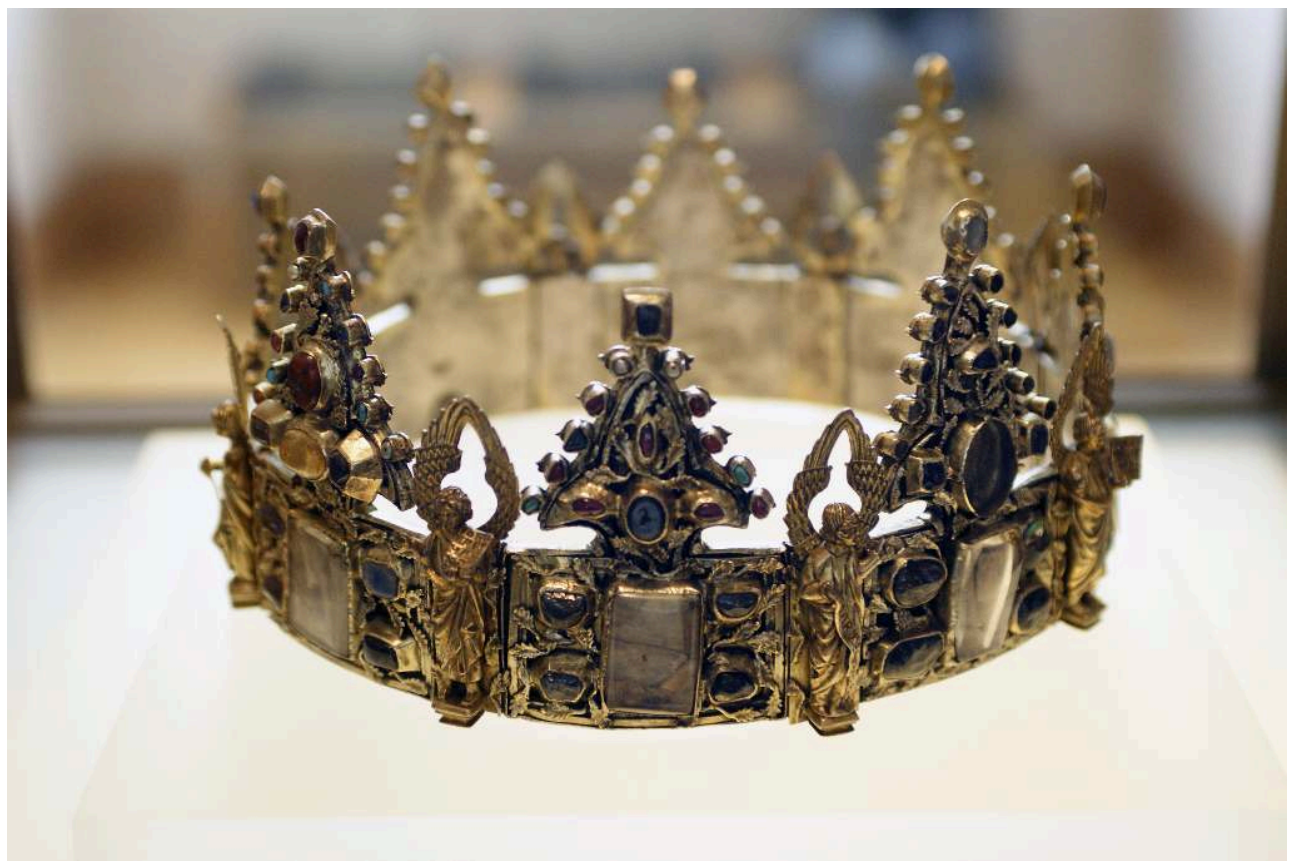

Couronne reliquaire dite "Couronne de Liège », XIIIe siècle, Paris, Musée du Louvre

11 Le second ensemble touche "l'ornement annulaire » (der Ringschmuck) et embrasse un très large éventail de parures : couronne, diadème, bandeau, collier, ceinture, brassard, bracelet, ourlet, bague... (fig. 6) « La spécificité de ces ornements tient au fait qu'ils sont généralement disposés sous forme d'arrangement périphérique ou périphérique-radial autour de l'objet décoré, qui occupe la position de cœur ou point central des relations qui structurent l'espace $»^{18}$.

Fig. 7

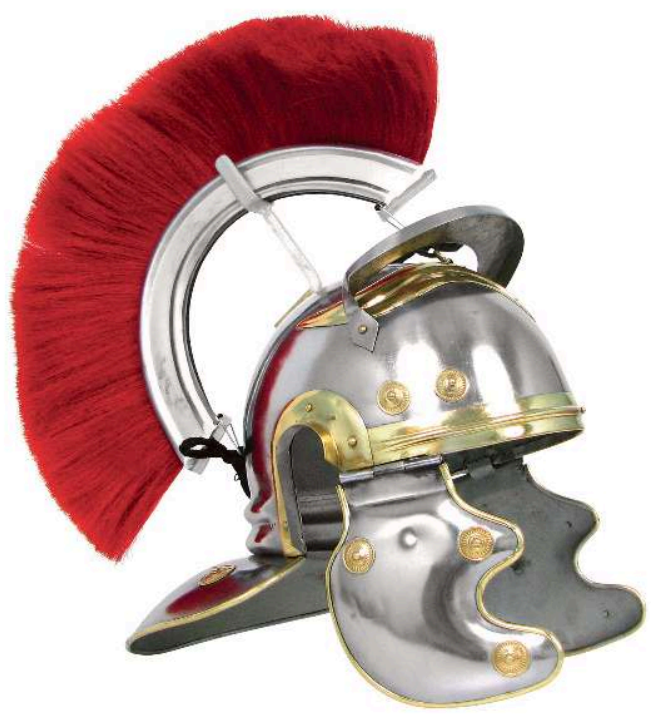

Casque romain « impérial-Gaulois » avec cimier, ler siècle ap. J.-C. (réplique contemporaine) 
Enfin, le dernier ensemble concerne « l'ornement directionnel » (der Richtungschmuck) : « cette espèce de parure qui sert à rehausser la direction et le mouvement du corps (...) qui renvoie principalement à l'opposition entre l'avant et l'arrière d'un phénomène, et joue de préférence sur la perspective de profil ${ }^{19}$. Ainsi le casque guerrier, encore plus lorsqu'il est garni d'un cimier, la chevelure souple laissée flottante, les vêtements amples se mouvant librement, les rubans (quand ils tombent) ou encore l'agrafe, la broche, etc. (fig. 7, 8).

Fig. 8

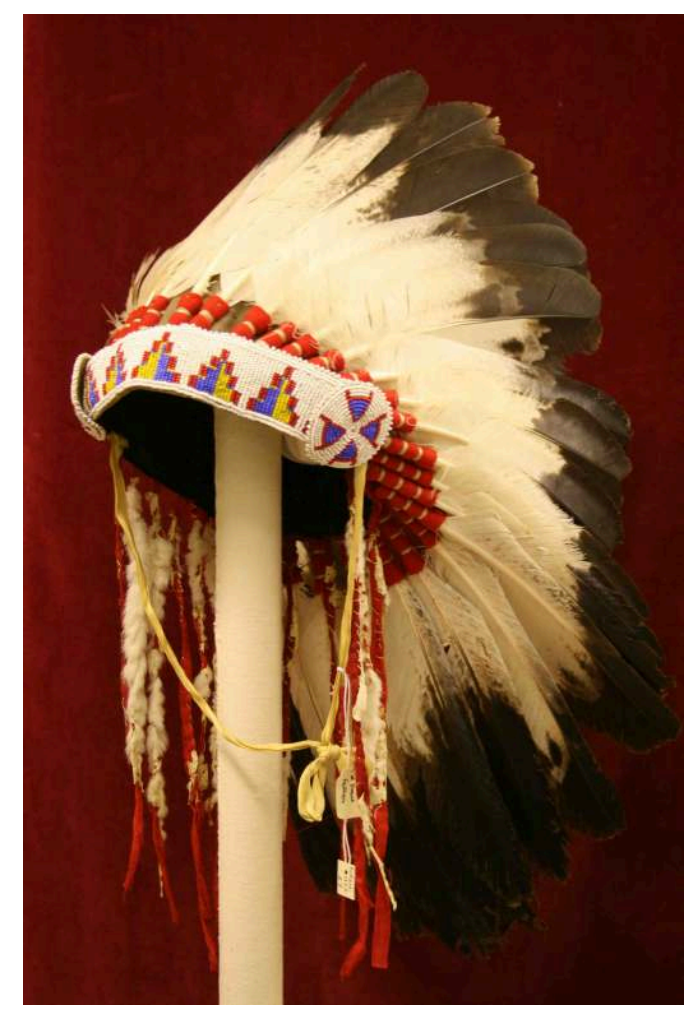

Bonnet de guerre Lakota, $X X^{e}$ siècle, Londres, Bristish Museum

«Pendant», « annulaire» et «directionnel» ne renvoient pas seulement à des catégories d'objets mais plus profondément à des orientations physiques ou des dimensions naturelles que Semper présentera de manière systématique dans les "Prolégomènes " à Der Stil, son opus major ${ }^{20}$. En effet, à la symétrie des ornements pendants correspondra (nous reviendrons bientôt sur ce mot de "correspondance ») une dimension verticale ou un axe de hauteur, tendu par la polarité physique et organique de la gravitation et de la croissance. Semper prendra ici modèle sur le monde végétal, et c'est la plante, "symétrique avant tout dans sa projection verticale ${ }^{21}$ qui donnera figure à un principe de symétrie. De la même manière, les ornements annulaires actualiseront une dimension toute en largeur que le théoricien nommera " proportionnalité »et qui trouvera sa figure naturelle dans le monde cristallin : «le principe de proportionnalité » (das Gesetz der Proportionalität) est déjà perceptible dans la forme radiale et fermée des cristaux, les rayons individuels apparaissant parfois articulés $»^{22}$. Enfin, les ornements directionnels détermineront une dimension de longueur ou de profondeur, selon un axe tendu entre inertie et volonté, entre pesanteur d'un côté et « force vitale » ou «force libre » volonté de l'autre ${ }^{23}$. 
Au travers de cette triple détermination ornementale, commente Jacques Soulillou, l'homme inscrit son activité créatrice au cœur du kosmos, concept clé de la théorie esthétique de Semper, en ce sens que se nouent en lui les notions d'ordre, de parure et de monde. Orner revient à reconnaître et à s'inscrire dans cet ordre cosmique, rendant visible les forces physiques sous-jacentes ${ }^{24}$.

\section{De l'unité cosmique à la loi naturelle}

Il est évident qu'une telle "physique », fût-elle articulée aux phénomènes esthétiques, ne résiste pas un instant aux avancées contemporaines des sciences physiques et au monde totalement renouvelé dont elles ont accouché dès la fin du XIX siècle, en réglant son compte à l'idée d'un cosmos orthonormé, simple et régulier ou mieux, en en finissant purement et simplement avec l'idée même de cosmos comme monde unifié. C'est bien pourquoi on dira fondamentalement grecque cette conception de la parure articulée à l'unité naturelle d'un cosmos organisé, ce que Semper au demeurant, revendiquait explicitement ${ }^{25}$.

Vision périmée? - voire. Pour datée qu'elle paraisse, une telle position théorique s'étend bien au-delà de la situation historique de son héraut, autant que du conservatisme de ses choix esthétiques. C'est à Ernst Gombrich qu'il sera revenu, dans son grand livre sur l'ornement - The Sense of Order, d'en donner une version moderne, modernisée ${ }^{26}$. À quoi tiendrait une telle modernité ? Au fait d'avoir expurgé l'idée d'ordre de la question de l'unité cosmique ou, pour le dire autrement, d'être passé du Cosmos à la Nature. À la lettre, la position de Gombrich doit être dite naturaliste. The Sense of Order: toute la thèse de Gombrich, en effet, reprend à son compte l'antique opposition de l'ordre et du désordre, du cosmos et du chaos : « [...] c'est le contraste entre le désordre et l'ordre qui alerte notre perception ${ }^{27}$. Mais si l'opposition ordre-désordre est conservée, ce n'est plus au titre d'une ordonnance physique générale du monde mais au titre de la pure légalité de ses règles et principes : l'ordre dit alors l'ensemble des lois de la nature ${ }^{28}$. La parure, et au-delà tout ensemble ornemental, obéit à des lois naturelles, dont l'ordre ne tient qu'à leur seule légalité et non à leur articulation en un tout unitaire. On dira donc que l'ornement est davantage naturel que cosmique. De fait, Gombrich prenait acte du tournant moderne par lequel la science, de Galilée à Newton, et la philosophie, de Descartes à Kant, avaient fait éclater le cosmos des Anciens, pour n'en saisir la totalité que par l'unité d'application de règles. Non sans hasard, The Sense of Order s'ouvre avec Kant ${ }^{29}$, ce même Kant qui aura critiqué la cosmologie rationnelle en plaçant l'unité naturelle non plus dans un monde objectif mais au cœur de la subjectivitée $e^{30}$. 
Fig. 9

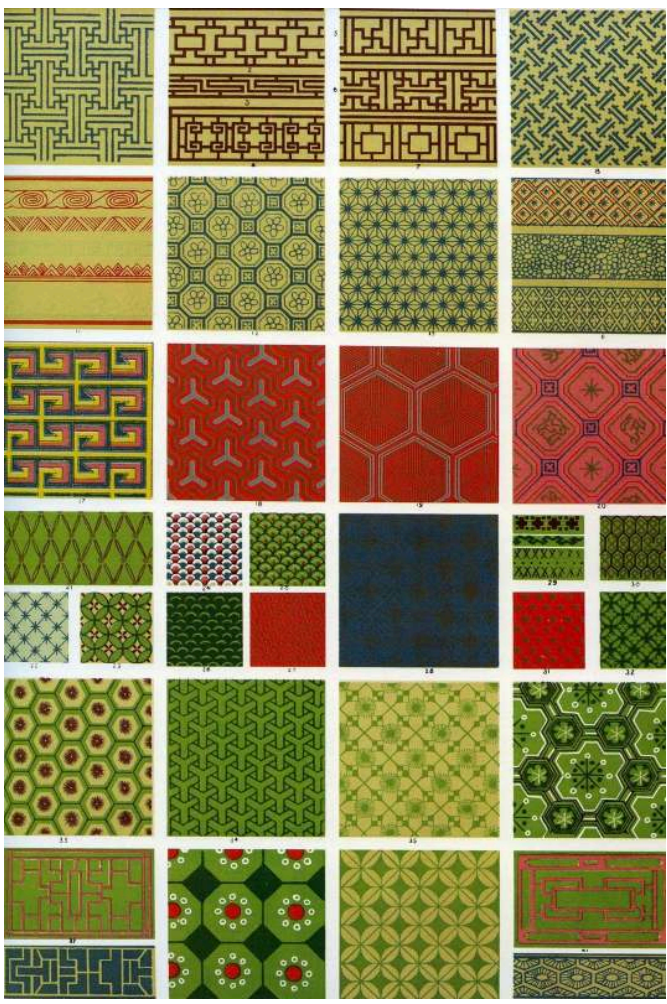

Ornements chinois, planche tirée de Owen Jones, The Grammar of Ornament, Londres, 1856

16 Or, quand on retire à l'ordre son unité ou sa cohérence, que reste-t-il ? - il reste des régularités (fig. 9). C'est donc tout logiquement que Gombrich tournait son regard presque exclusivement sur les formes simples et régulières, autant dire sur les formes géométriques pour inscrire l'ornemental dans un ordre naturel : « la création d'ordres se fonde sur les lois de la géométrie $»^{31}$ - façon de retrouver ce vieux fonds pythagoricien qui était à l'œuvre dans toute pensée de l'ordre cosmique. De là le profond géométrisme de Gombrich, qui ne semble vouloir connaître d'ornement que damiers, grilles, spirales et symétries, toujours réductibles à quelques figures régulières, le plus souvent le cercle et le carré, et qui ne peut que reléguer les marginalia médiévales, les grotesques renaissants ou les volutes rococo « au bord du chaos $»^{32}$ (fig. 10). 


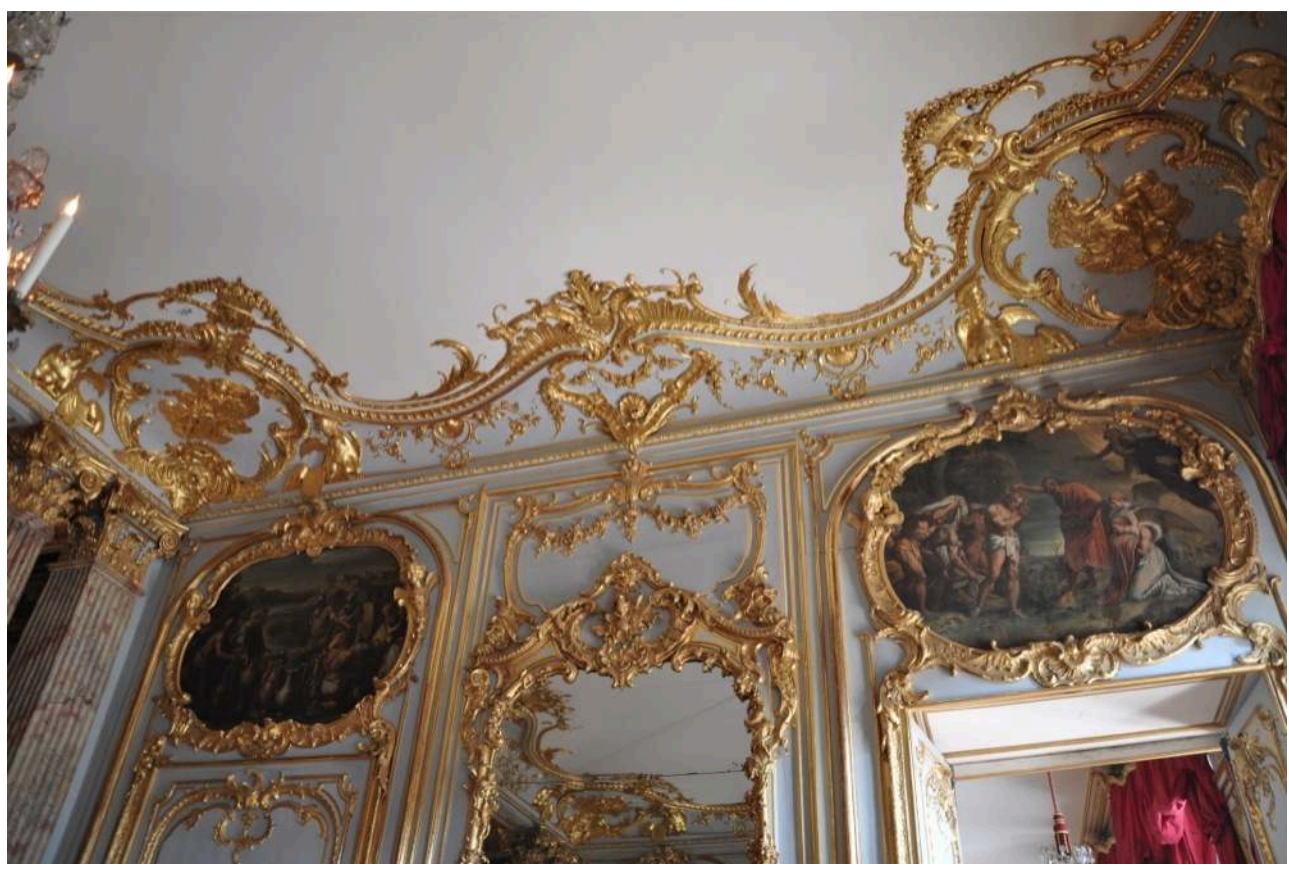

Décoration rocaille, XVIII siècle, Palais Rohan, Strasbourg

17 Mais dans le fond, il appert vite que la régularité elle-même peut très bien se passer de la figure étendue, fût-elle géométrique, fût-elle régulière, pour ne consister qu'en de pures règles logiques. C'est donc fondamentalement sous la forme d'une sorte de table des catégories que pourra se penser l'ordre ornemental et ses possibilités naturelles. Les principes d'une telle table n'ont eux-mêmes rien d'ornemental. Quand Charles Blanc, par exemple, dans son traité de 1875 - L'Art dans la parure et dans le vêtement distingue cinq principes fondamentaux dont l'application engendre «les motifs sans nombre que les hommes ont inventés jusqu'à ce jour et ceux qu'ils inventeront encore pour orner leurs personnes, leurs demeures ou leurs temples ", à savoir, « la répétition, l'alternance, la symétrie, la progression, la confusion $»^{33}$, la constitution d'un ordre ne tient qu'à des relations logiques entre des termes dont la nature, dans le fond, importe peu. C'est bien du reste cette naturalité des principes, leur nature logique pour le dire vite, qui leur donne une légalité universelle: les phénomènes de répétition, de progression, de symétrie, de rayonnement, de gradation, etc., se rencontrent à tous les niveaux de l'univers, dans toutes les strates cosmiques, matérielle, organique, symbolique, humaine, animale, microscopique, macroscopique, etc. En sorte que l'ornemental donnera le paradigme d'une mathesis de l'ordre universel, une mathesis qui trouvera sa figure dans l'ornement, mais qui pourra se dire dans n'importe quel langage formulaire : ainsi, par exemple, des tables de permutation de Dominique Douat, dans sa Méthode pour faire une infinité de desseins différents avec des carreaux mi-partis de deux couleurs par une ligne diagonale de 1722, qui trouvent leur chiffre dans la permutation des quatre premières lettres de l'alphabet (fig. 11) ${ }^{34}$. 
Fig. 11

\begin{tabular}{|c|c|c|c|}
\hline \multicolumn{4}{|c|}{$\begin{array}{l}\text { Continuation dela Tabie } \\
\text { de256 Pernutations. }\end{array}$} \\
\hline${ }_{20}$ & ${ }_{445} \backslash \mathrm{N}$ & $e_{x} \backslash \Delta \mathbf{D}$ & ${ }_{777} \times / \Lambda$ \\
\hline${ }_{130} \nabla$ & ${ }_{146} \backslash \mathrm{C} \backslash$ & $162 \mathrm{C}=\mathrm{V}$ & ${ }_{78}^{78} \backslash \Lambda$ \\
\hline${ }^{3} \times V^{\prime}$ & ${ }_{47} \mathbf{Q C V}$ & ${ }^{6} \sigma_{3} \backslash \backslash$ & $179 \mathrm{SWC}$ \\
\hline${ }_{132}$ खण & $48 \square \nabla$ & $.604 \backslash$ & $s c \square$ \\
\hline $33 \mathrm{WV}$ & ${ }_{49} \backslash \boldsymbol{\lambda}$ & ${ }_{26} \backslash \backslash \backslash$ & ${ }_{g s} \backslash \mathbf{D}$ \\
\hline${ }_{34} \backslash \backslash$ & $250 D V$ & $.66 \mathrm{TWN}$ & ${ }_{g}>\mathrm{Da}$ \\
\hline${ }_{35} \nabla V \nabla$ & ${ }^{2}{ }^{\prime \prime} \nabla \boldsymbol{\Delta}_{\Delta}$ & ${ }^{6} 6_{7} \mathrm{NV}$ & ${ }_{83} \times$ \\
\hline${ }_{3}^{6} 6 \nabla \nabla$ & ${ }_{152} \nabla / 1$ & $1 6 8 \longdiv { \square A C }$ & ${ }_{8+10}$ \\
\hline $137 \backslash \backslash \nabla$ & 's3 $\nabla V \square$ & $\operatorname{sig} \backslash \mathbf{V}$ & 85 प्a \\
\hline${ }_{3 e} \nabla \boldsymbol{V}$ & ${ }^{*} \mathrm{DV}$ & $170 \backslash \mathrm{M} / \mathrm{a}$ & $y^{6} \times 1$ \\
\hline $139 \mathbf{X N}$ & ${ }_{55} \triangle \nabla$ & 171 & ${ }_{87} D V$ \\
\hline $40 D /$ & ${ }_{15^{6}} \backslash \sqrt{ }$ & ${ }_{172} \backslash \mathrm{V}$ & ${ }^{188}$ W6 \\
\hline 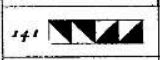 & ${ }_{157} \mathrm{~W}$ & $13 \square \mathrm{CA}$ & ${ }_{180} \mathbf{C D}$ \\
\hline${ }^{1+2} \square$ & ${ }_{15 g} \backslash \bar{\square}$ & $174 \nabla \mathbf{Q V}$ & $190 \mathrm{VN}$ \\
\hline${ }^{4} 3 \Delta \Delta$ & ${ }_{60} \square \square$ & ${ }_{775} \backslash \mathbf{D}$ & $\operatorname{tg} D$ \\
\hline${ }_{44} \nabla \nabla$ & $160 \triangle V /$ & ${ }_{176} \backslash \mathbf{W}$ & $\lg ^{2} \backslash \mathrm{V}$ \\
\hline
\end{tabular}

Table de permutations, planche tirée de D. Douat, Méthode pour faire une infinité de desseins différens avec des carreaux mi-partis de deux couleurs par une ligne diagonale, Paris, 1722

\section{Critique de l'analogisme : quelle unité cosmique?}

On touche ici, sans aucun doute, le fond critique du problème : du diagramme de Semper à la table de Blanc aux analyses de Gombrich, il s'agit toujours de penser la rapport de la parure au monde sur un mode analogique. Dans tous les cas de figure présentés, c'est toujours par analogie que la cosmétique est cosmique. Encore une fois, le principe a eu beau être clairement exprimé par les Grecs, il est à l'œuvre en bien des contrées de la planète. C'est par analogie, entre autres mille exemples, que les Kayapo d'Amazonie associent leur grande parure dorsale krokrokti à l'astre solaire (fig. 12) : les centaines de plumes caudales d'ara qui la composent sont montées selon une disposition radiale analogue aux rayons du soleil ${ }^{35}$. 
Fig. 12

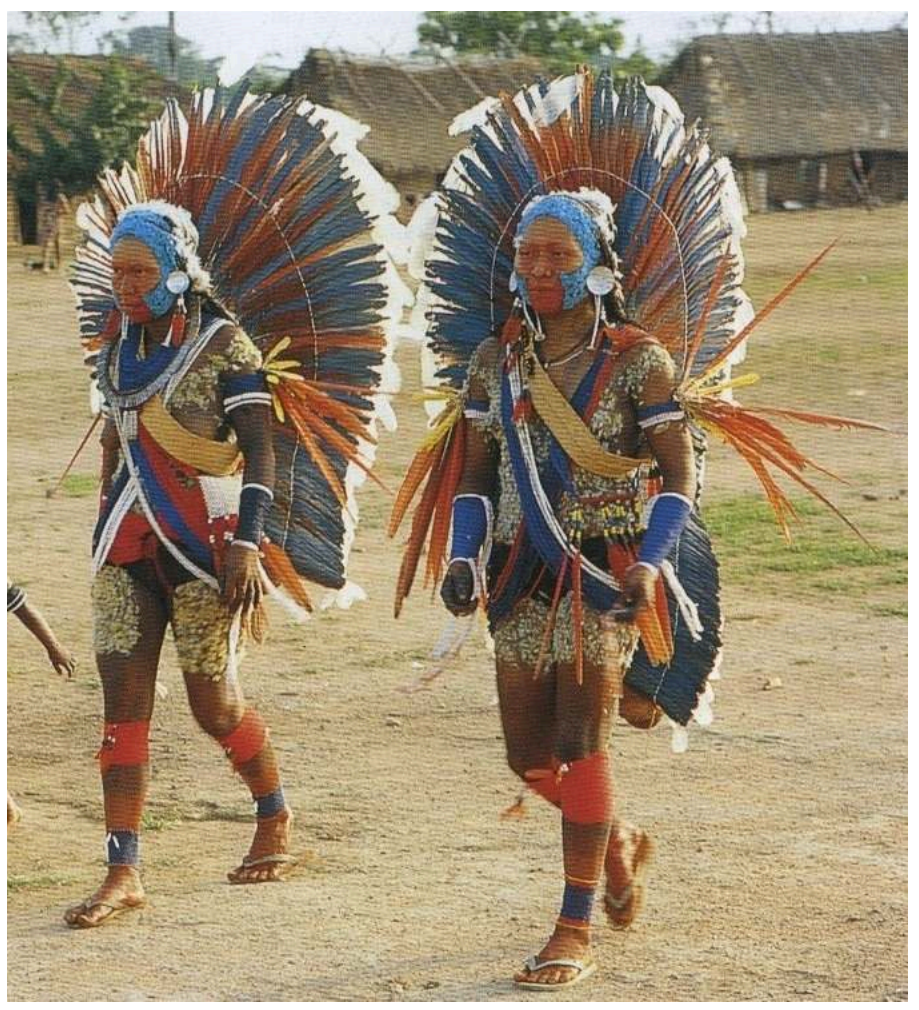

Femmes Kayapo portant le krokrokti, Brésil, Para, photo G. Verswijver

19 Pourtant, aussi prégnant soit-il, historiquement, anthropologiquement, philosophiquement, un tel modèle ne peut que se montrer insuffisant voire illusoire. On sait que l'analogie, dans son acception la plus simple, mais aussi la plus efficace, repose sur une ressemblance de proportion: $\mathrm{A}$ est à $\mathrm{B}$ ce que $\mathrm{C}$ est à $\mathrm{D}$. Le modèle analogique permet ainsi de penser la mondanité d'une parure dans le rapport entre deux ordres ${ }^{36}$. Autant dire que la continuité entre les deux n'est pas réelle mais ne tient qu'en une représentation, et donc que, dans le fond, le cosmétique n'est cosmique que par une vue de l'esprit: il faut toujours un troisième terme commun, une médiation, pour que l'analogie puisse tenir et que s'établisse la correspondance entre les deux ordres. C'est bien cette vue de l'esprit qui impose à la parure comme au monde un lourd prix à payer : celui d'une idéalisation, autrement dit d'une simplification. Il aura en effet fallu simplifier la parure, la réduire à des formes régulières, géométriques le plus souvent, autant qu'à une forme globale pour l'attacher à l'ordre du monde. Mais quel ornement corporel se réduit à un simple cercle, à un simple plan régulier (pour ne rien dire de la mise en mouvement de la parure par son port, et bien que Semper ait tenu compte de cette dimension dynamique) ? À partir de là, toutes les parures se ressemblent à peu près et l'incomparable variété des formes cosmétiques se retrouve très vite réduite à un catalogue à trois ou quatre entrées. En attachant analogiquement la régularité cosmétique à la régularité cosmique, c'est bien la singularité de la parure en général, autant que la singularité de cette parure singulière qui se dissout.

On aurait pourtant tort de croire que la singularité est inversement proportionnelle à l'ouverture cosmique de la parure. Non qu'il n'y ait pas de régularités dans la cosmétique, mais ce n'est assurément pas par elles que se signale sa dimension cosmique. Car il s'agit d'abord de penser une mondanité de la parure qui soit réelle; il 
s'agit d'éprouver un rapport du monde et de l'ornement corporel qui ne passe plus par la médiation d'une analogie mais s'impose dans sa souveraine continuité. La voie qui se dessine prend ainsi un double contre-pied. Elle contrevient d'abord à l'idée antique (et largement répandue sur la planète) d'un ordre cosmique conçu comme bel assemblage. En cela, elle s'inscrit parfaitement dans la modernité des XVII ${ }^{e}$ et XVIII ${ }^{e}$ siècles et sa critique du cosmos comme univers $\operatorname{clos}^{37}$. "Le monde n'a pas de contenus signifiants d'après lesquels on pourrait le systématiser, ni de significations idéales d'après lesquelles on pourrait l'ordonner, le hiérarchiser $"^{38}$. Mais elle contrevient tout autant à cette même modernité et à son leitmotiv de la "perte du monde", en considérant notamment comme nulle et non advenue la critique kantienne de la cosmologie rationnelle. De Nietzsche à Deleuze, en passant par Whitehead, Bergson et d'autres encore, c'est tout un pan (certes minoritaire) de la métaphysique occidentale, une " autre métaphysique ${ }^{39}$, qui a d'un même geste refusé la subjectivisation moderne du monde sans pour autant revenir à l'objectivité grecque d'un ordre transcendant. A la rigueur, on pourrait dire que notre tâche est l'exacte inverse de celle que s'était fixée Ernst Gombrich: tandis qu'en moderne naturaliste, l'historien de l'art expurgeait l'ordre (ornemental et naturel) de toute unité cosmique, il conviendrait d'expurger l'unité cosmique de toute idée d'ordre, si tant est qu'une telle " unité » doive dorénavant moins se penser comme cosmos, l'idée d'ordre lui restant peut-être irrémédiablement attachée, que, pour parler comme Joyce, chaosmos.

\section{Les affres de la projection}

21 Toute cette belle métaphysique restera pourtant un vœux pieux tant que l'on n'aura pas posé la question cruciale de savoir comment se détermine morphologiquement la cosmicité de la parure. Quelles sont les formes d'un devenir-monde des ornements corporels? La question ne supporte évidemment pas de réponse directe mais suppose d'en revenir à d'autres questions venant chatouiller quelques évidences sur ce qu'est une parure ${ }^{40}$.

Il est tout à fait significatif que dans son Manuel d'ethnographie, Marcel Mauss ait voulu diviser dans le concept de cosmétique, jugeant peut-être son unité esthétique factice. L'ethnologue distinguait ainsi entre «la cosmétique » à proprement parler, conçue comme «ornementique directe du corps» et la parure conçue elle comme "ornementique indirecte du corps $»^{41}$. Tandis que la première prend directement le corps pour objet plastique et recouvre grosso modo toutes les pratiques d'arrangement et de défiguration corporels (de la coiffure aux scarifications, des déformations crâniennes aux tatouages, de toutes les formes d'épilation aux perforations nasales, etc. $)^{42}$, la parure consiste pour sa part en « l'addition d'ornements au corps » et s'étend du vêtement à tous les types de coiffes, en passant par les masques, les bijoux, etc. ${ }^{43}$. Ce partage est très significatif dans la mesure où, pour reprendre les termes de Mauss, c'est l'ornementique qui prête à la cosmétique son trait fondamental : sa nature d'ajout, d'élément rapporté. Dans les deux en effet, le corps est toujours conçu comme le support d'un ajout extérieur: un objet, aux matières et formes innombrables, pour l'ornementique, tandis que pour la cosmétique, c'est "la beauté » elle-même qui fait office d'objet extérieur ${ }^{44}$. Il est dans le fond très étonnant que cette distinction qui semblait découler du sens commun au premier abord, en arrive à nier l'évidence sensible: qu'est-ce donc qui peut bien s'ajouter au corps dans l'arrangement des 
cheveux, dans l'épilation des sourcils, dans une déformation ${ }^{45}$ ? Matériellement, rien. Mais pourtant, l'anthropologue aura vite interprété cet ajout comme une marque signifiante, autrement dit comme un objet symbolique. La parure, qu'elle soit d'arrangement ou d'objet, fonctionne ainsi comme une inscription sociale ou subjective, donnant au corps une seconde peau. On appellera projectif un tel modèle cosmétique, pour dire le rapport d'adéquation entre le paré et la parure, entre un corps intérieur servant de support, ou mieux: de subjectile, pour la projection d'un corps extérieur. Cette projection, très souvent, peut aller jusqu'à l'incorporation ${ }^{46}$ : l'extériorité de la parure est comme intériorisée par le corps, avec tous les accents dramatiques que l'homme moderne aura voulu voir dans cette "extension tragique " d'un corps dans ses atours, étrangers à sa substance, pour parler comme Aby Warburg ${ }^{47}$. Et si l'on refuse aux animaux la parure (en Occident, s'entend), c'est bien parce que les animaux ne projettent rien. Ils ont des habitudes, mais point d'habits. Mieux : si le vêtement, au moins dans toute la tradition antique et chrétienne, est tenu pour le propre de l'homme, c'est parce que la projection humaine aura trouvé dans le vêtement ou plus exactement dans le revêtement quelque chose comme son acte originaire, comme sa dynamique souveraine : un corps second et culturel - habits, parures, objets - s'applique à un corps premier, nu et naturel ${ }^{48}$.

Fig. 13

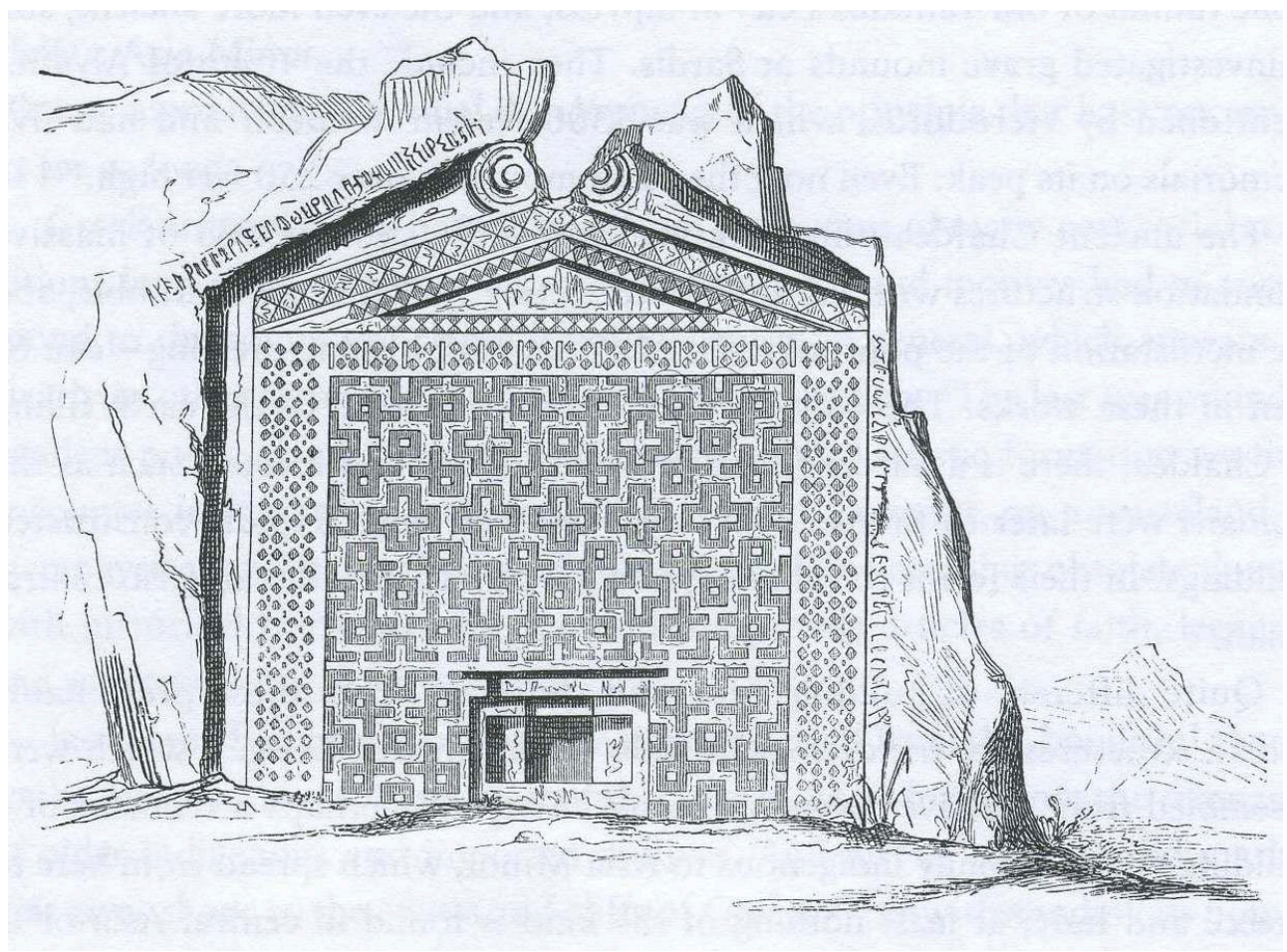

Façade de la tombe de Midas, planche tirée de G. Semper, Der Stil, Munich, 1860

Jacques Soulillou a fourni quelques jalons pour critiquer un tel modèle projectif en montant une dialectique de la vêture et de la parure. Ce faisant, c'est l'hypothèse de Gottfried Semper qui se trouve revivifiée, soit cette sorte de fiction historique voulant que tous les arts, à commencer par l'architecture, tirent leur origine de la fabrication des textiles - non plus, donc, le Semper penseur de l'architecture cosmique, mais le Semper généalogiste du tissage technique et esthétique ${ }^{49}$ (fig. 13). Le principe de 
l'habillement ou du revêtement (Bekleidung) désignait en effet pour Semper tout le contraire d'un acte projectif, dans la mesure où le vêtement lui-même n'était pas à ses yeux un être de surface plaqué sur un corps. Le vêtement était d'emblée considéré comme un élément plastique ayant une valeur propre: le vêtement comme parure. Ainsi le tissage sous forme de clôture délimitant un espace a sans aucun doute précédé la construction de murs. En sorte que l'ornement est structurel par essence (si le tressage d'une cloison est considéré comme un motif), et ce n'est qu'après coup, historiquement, qu'il s'est développé dans une différenciation, sinon une opposition avec la structure (un placage). C'est donc la totalité de l'édifice qui doit se penser comme parure, dans la mesure où elle fait corps avec lui : " la parure colle à la peau, alors même que le vêtement n'en est qu'une seconde dont l'interchangeabilité dénonce la destination abstraite pour un 'corps en général' ${ }^{50}$. La dialectique de la parure et du vêtement est celle entre une "adéquation entre la forme et la destination " et une " rupture de cette unité $»^{51}$.

Mais que signifie, précisément, "faire corps »? et comment passer de l'ordre architectural à l'ordre de l'ornement corporel ? Adaptation, union, adéquation... : dans tous les cas de figure, on voit qu'il s'agit au mieux de reconduire un très vieux schème hylémorphique où la matière épouse la forme, au pire de donner dans une sorte de synthèse idéale, pour ne pas dire d'union mystique entre corps et parure. La malédiction du vêtement et de la parure tient précisément à ce que l'on a voulu en faire l'histoire d'un véritable corps à corps, l'histoire de ces échanges entre corps organique et corps de parure : tantôt c'est la parure qui épouse les contours organiques, tantôt c'est le corps qui est sommé de s'adapter aux formes les plus contraignantes ${ }^{52}$ (on sait que la très forte contrition $\mathrm{du}$ corset féminin a pendant plusieurs siècles façonné profondément des tailles démesurément étroites). Pour que ces chocs aient lieu, il faut au préalable avoir "corporéisé » la parure, l'avoir transformée en un corps extensif divisible en parties subsumables en un tout ${ }^{53}$. Partes extra partes: le corps et ses atours s'ajoutent comme autant de parties à côté d'autres parties. En sorte qu'on ne peut voir entre eux qu'un rapport de contiguité, se rendant incapable par la suite de penser leur continuité, et au-delà, la continuité entre corps et monde.

\section{Impersonnel, incorporel, anorganique : devenir-monde}


Fig. 14

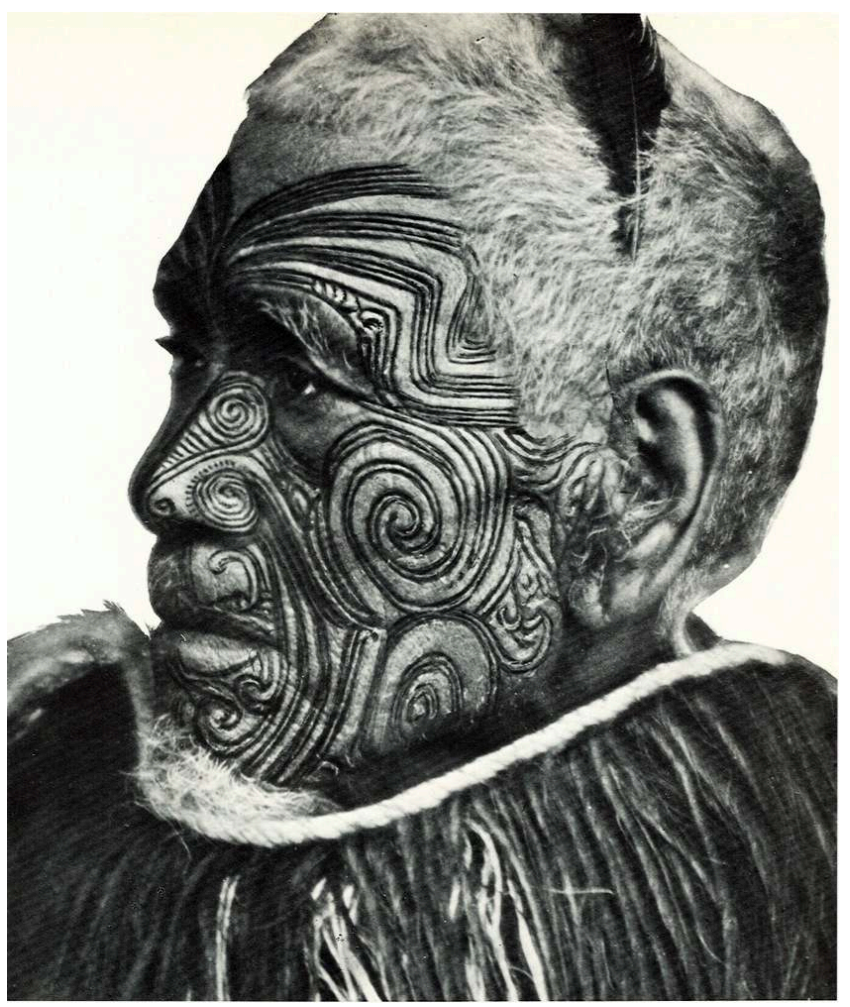

Tatouage Maori, photographie anonyme, XIXe siècle

On doit pourtant à Georg Simmel, dans un texte aussi bref qu'efficace, d'avoir magistralement cassé ce principe d'adéquation, en montrant que le ressort esthétique de l'élégance tenait précisément à une non-adéquation entre corps et parure. En posant que la parure est d'autant plus élégante qu'elle ne colle pas au corps, Simmel s'engouffrait dans cela même qu'aura dénoncé Jacques Soulillou comme une "destination abstraite pour un 'corps en général' " ${ }^{54}$ c'est "par l'intermédiaire d'un trait d'impersonnalité » que la parure gagne en intensité. Le tour de force de Simmel est du reste d'avoir placé le questionnement sur un terrain morphologique, en prêtant toute son attention à la modulation du rapport d'inadéquation entre la forme de l'ornement et la forme du corps orné (fig. 14, 15, 16) : 
Fig. 15

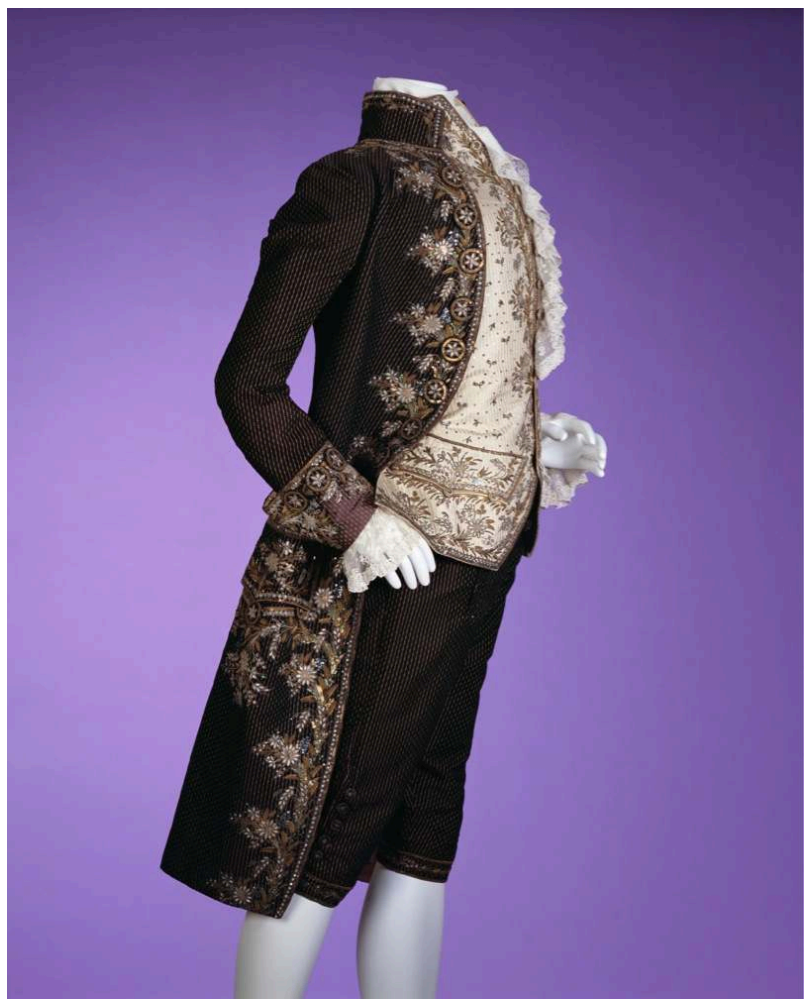

Habit de cour «à la française », vers 1780, Londres, Victoria and Albert Museum

Tout ce qui, de façon générale, 'pare' l'être humain, prend place sur une échelle de degrés, selon la façon plus ou moins étroite dont la parure est liée à la personnalité physique. La parure la plus étroitement liée au corps est caractéristique des peuplades primitives : c'est le tatouage. L'extrême opposé, c'est la parure de métal ou de pierre, qui n'est absolument pas liée à un individu et que chacun peut mettre. Entre les deux, il y a le vêtement - qui certes n'est pas aussi inéchangeable et personnel que le tatouage, mais qui est tout de même plus lié à l'individu et moins détachable de lui que la "parure" à proprement parler. Mais c'est précisément dans l'impersonnalité de celle-ci que réside son élégance. Alors que la pierre et le métal sont entièrement clos sur eux-mêmes, ne désignant aucune individualité précise et non modifiables du fait de leur dureté, ils sont malgré tout forcés de servir une personnalité : là est précisément l'attrait le plus subtil de la parure. L'élégance véritable évite l'individualisation extrême, elle instaure toujours une sphère de généralité, de stylisation, pour ainsi dire d'abstraction autour de l'individu - ce qui n'empêche évidemment pas les raffinements par lesquels ce caractère général se lie à la personnalité ${ }^{55}$. 
Fig. 16

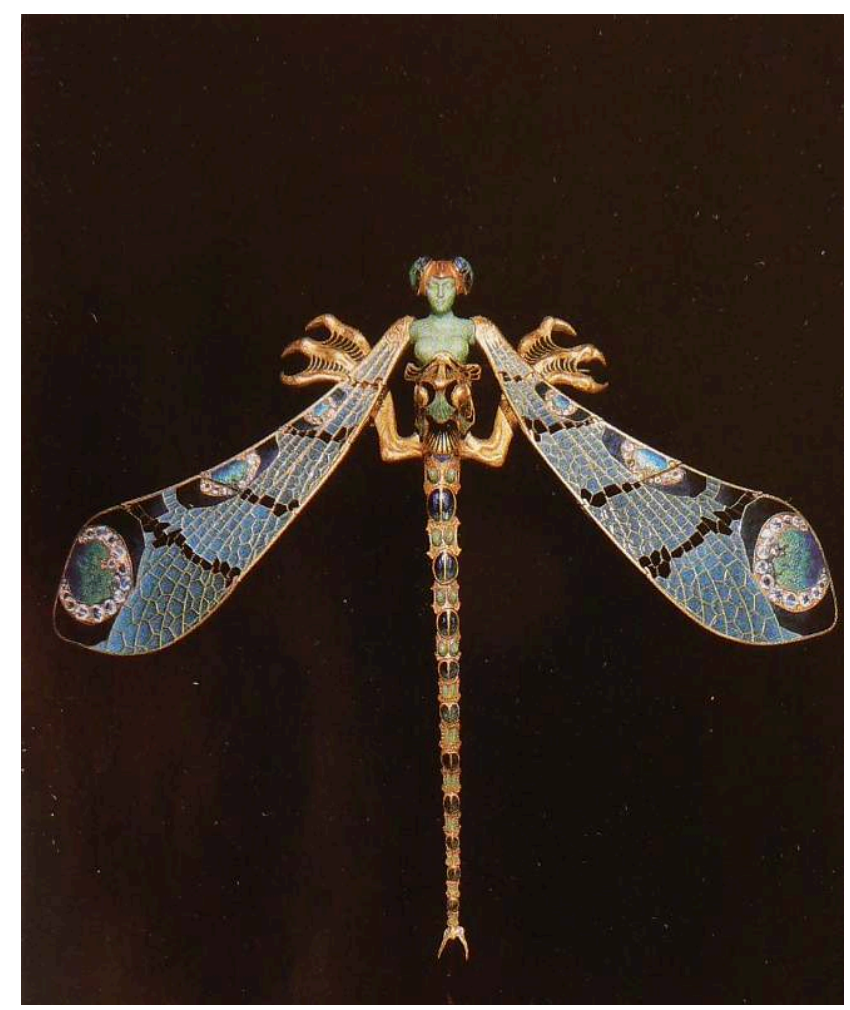

René Lalique, Pectoral à la libellule, vers 1900, Lisbonne, Fondation Calouste-Gulbenkian

Qu'est-ce que Simmel entend ici par «impersonnalité »de la parure, sinon le mouvement d'une décorporation? Parler de "parure abstraite » signifie alors qu'elle n'entre dans aucune relation mimétique ou d'imitation formelle avec le corps orné, mieux, qu'elle gagne en élégance ce qu'elle perd en ressemblance avec ce qu'elle pare. Une telle abstraction dit l'autonomie d'une parure qui n'a besoin d'aucun support pour exister. À la lettre, on dira que la parure est incorporelle, au sens exact où les Stoïciens ont pensé l'incorporel comme pur effet, ni actif, ni passif comme les corps, mais se produisant à la surface des corps dans une sobre neutralitée ${ }^{56}$. La parure n'existe donc pas comme les corps; elle subsiste à leur surface, toujours autonome et extérieure. Autant dire que la parure ne s'incorpore pas, comme le voudrait constamment le fantasme occidental, mais décorpore le corps. Jean-Clet Martin a très bien insisté sur ce « charme incorporel » de la parure qui expose le corps parfois jusqu'à l'extase ${ }^{57}$. Et s'il y a une " grammaire de la parure » (comme on parle d'une « grammaire de l'ornement »), il faudra dire que la parure n'est ni substantif (corps ou chose), ni adjectif (qualité) mais toujours verbe infinitif : elle ne qualifie ni ne substantialise le corps, elle l'infinitise. Il nous est sans doute très difficile de saisir une telle nature incorporelle, attachés que nous sommes aux propriétés objectives des choses autant qu'à leurs qualités formelles et matérielles. Parler d'une parure à l'infinitif, poser que la parure infinitise le corps, c'est regarder ses atours comme autant de motifs abstraits qui le virtualisent, si tant est que «le vêtement humain est une coupure à l'intérieur de l'homme, non pas entre le corps et l'extérieur, mais entre le corps anatomique et un corps prothétique et purement virtuel $\aleph^{58}$.

Or, ce sont ces événements incorporels qui vouent les parures à un destin cosmique, qui tracent une continuité réelle avec le monde. On a dès lors quitté le régime des chocs, ce 
fracas extensif entre corps et parure, pour pénétrer dans le réseau des continuités cosmiques, des devenirs-monde. "Dans toute cosmétique destinée à souligner notre individualité, écrit très justement Emanuele Coccia dans le prolongement des analyses de Simmel, nous nous confondons avec un trait du monde (un peu de poudre colorée, quelque pierre, du métal particulier, un morceau d'étoffe bien coupé) qui n'a aucun rapport avec nous (ni selon l'être, ni selon la génération, ni selon la forme, ni selon la matière) $\aleph^{59}$. Cette continuité ne tient donc pas simplement à une question de matière mais bien plus profondément à la continuité d'un trait. Ce qui donne ses coordonnées cosmiques à la parure, c'est sa façon très particulière de réduire le corps, de le concentrer en quelques lignes, motifs, couleurs, en un mot et à la lettre : de l'abstraire. Gilles Deleuze et Félix Guattari auront nommé « ligne abstraite » cette continuité entre moi et le monde qui ne passe qu'au prix d'une dépersonnalisation, d'une abstraction où le corps se stylise :

À force d'éliminer, on n'est plus qu'une ligne abstraite, ou bien une pièce de puzzle en elle-même abstraite. Et c'est en conjuguant, en continuant avec d'autres lignes, d'autres pièces qu'on fait un monde, qui pourrait recouvrir le premier comme en transparence. L'élégance animale, le poisson-camoufleur, le clandestin: il est parcouru de lignes abstraites qui ne ressemblent à rien, et qui ne suivent même pas ses divisions organiques ; mais ainsi désorganisé, désarticulé, il fait monde avec les lignes d'un rocher, du sable et des plantes, pour devenir imperceptible. Le poisson est comme le peintre poète chinois : ni imitatif, ni structural, mais cosmique. [...] Le cosmos comme machine abstraite, et chaque monde comme agencement concret qui l'effectue. Se réduire à une ou plusieurs lignes abstraites qui vont se continuer et se conjuguer avec d'autres, pour produire immédiatement, directement, un monde, dans lequel c'est le monde qui devient, on devient tout le monde. [...] Être à l'heure du monde. Voilà le lien entre imperceptible, indiscernable, impersonnel, les trois vertus ${ }^{60}$.

L'exubérance des parures les plus protubérantes, l'extravagance des atours les plus bigarrés, tout cela est paradoxalement le corrélat d'un devenir-imperceptible où l'on se fond dans le monde, où l'on fait monde. "L'imperceptible est la fin immanente du devenir, sa formule cosmique ${ }^{61}$. L'imperceptibilité en question ne tient qu'à l'abstraction de la parure qui se retire, s'abstrait des catégories discontinues où la perception claire et distincte veut la ranger, en venant neutraliser ses oppositions tranchées. 


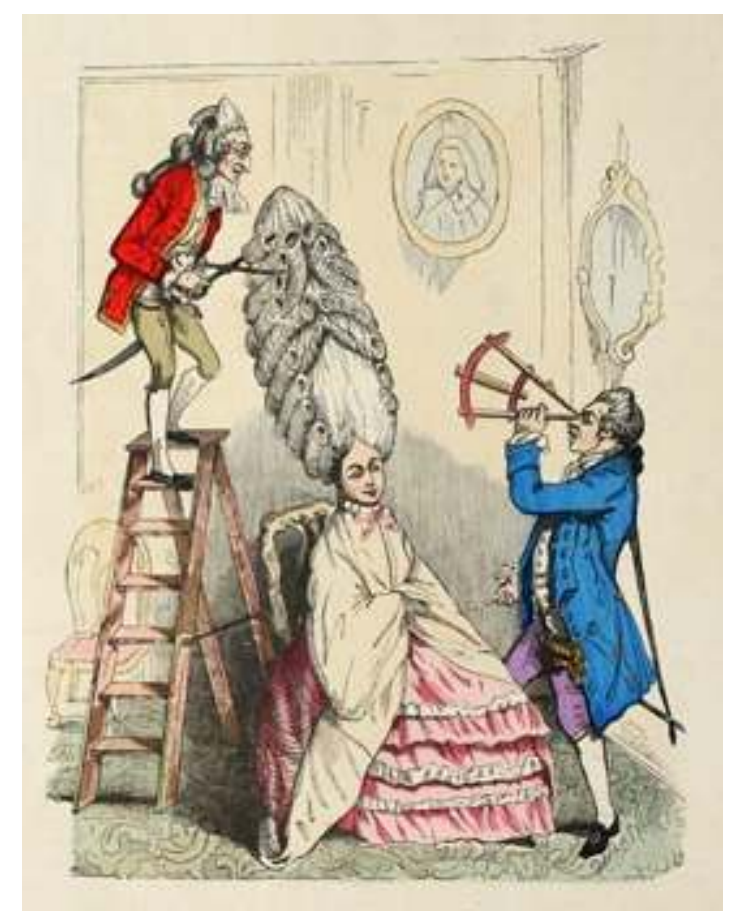

M. Darly, Coiffure à l'échelle, vers 1770, gravure

Il n'y a donc plus à dramatiser comme le faisait Warburg le hiatus entre le corps propre organique et la parure ou le vêtement inorganique. Les cris et les larmes de cette « tragédie » ne sont-ils pas feints ? - en ce sens qu'il n'y aurait de hiatus qu'à tenir pour valable l'opposition frontale de l'organique et de l'inorganique. Mais l'efficace de la parure est précisément de se déployer dans un intervalle qui n'est ni organique ni inorganique mais anorganique. L'anorganique, pour reprendre le vocabulaire de Gilles Deleuze et Félix Guattari, dit magistralement et très concrètement ce qu'il faut entendre par "parure abstraite». Les perruques féminines de la seconde moitié du $\mathrm{XVIII}^{\mathrm{e}}$ siècle sont connues pour leur extravagance, que les contemporains n'ont pas manqué de railler. Pouvant atteindre des dimensions démesurées, elles s'ornaient de plumes, de perles, de rubans, voire de modèles réduits de bateaux : déjà tout un monde (fig. 17). Mais qu'est-ce qu'une perruque, à la différence du simple postiche ${ }^{62}$, sinon un chapeau de cheveux? La distinction entre la coiffe et la coiffure s'y trouve neutralisée, pour laisser place à un impersonnelle et anorganique trait capillaire. 
Fig. 18

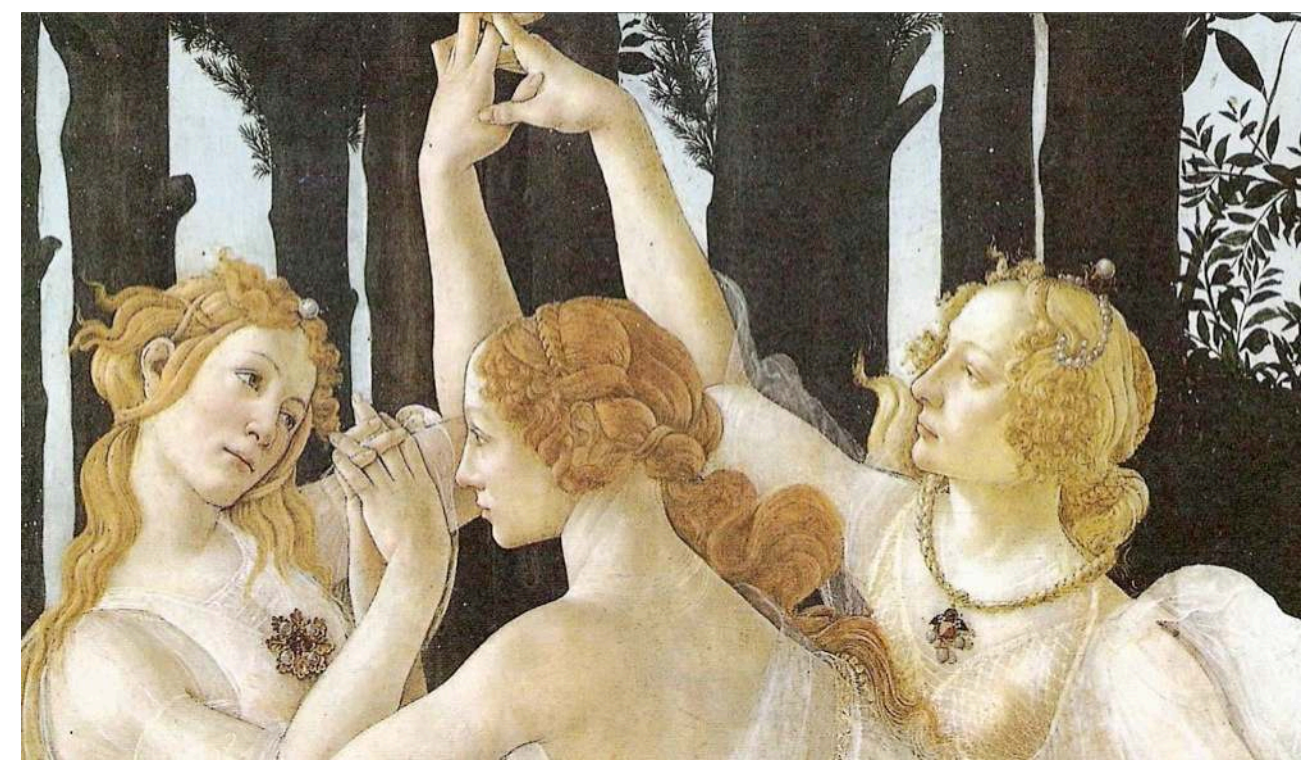

S. Botticelli, Le Printemps (détail), ca. 1481-1482, Florence, Galerie des Offices

La perruque n'est en effet pas tout à fait inorganique, puisqu'elle est faite de cheveux et apposée sur mon corps ; mais elle n'est pas tout à fait organique puisqu'elle n'est pas faite avec mes cheveux, qu'elle ne respecte pas la couleur naturelle de ma pilosité, et surtout, puisqu'elle ne suit plus du tout les contours de ma boîte crânienne et se développe davantage comme une protubérance. Elle fait ainsi se lever une chevelurecoiffure abstraite existant en soi, distincte du corps propre, indépendante de toute forme et matière organique. Les improbables coiffures des Trois Grâces que peint Botticelli dans Le Printemps, ou que l'on trouve dans toutes ces « Têtes idéales » de la fin $\mathrm{du}$ Quattrocento florentin (fig. 18, 19), n'ont pas d'autre but que faire se décoller une manière capillaire, que de faire se lever une coiffure distincte en droit de toute chevelure. 
Fig. 19

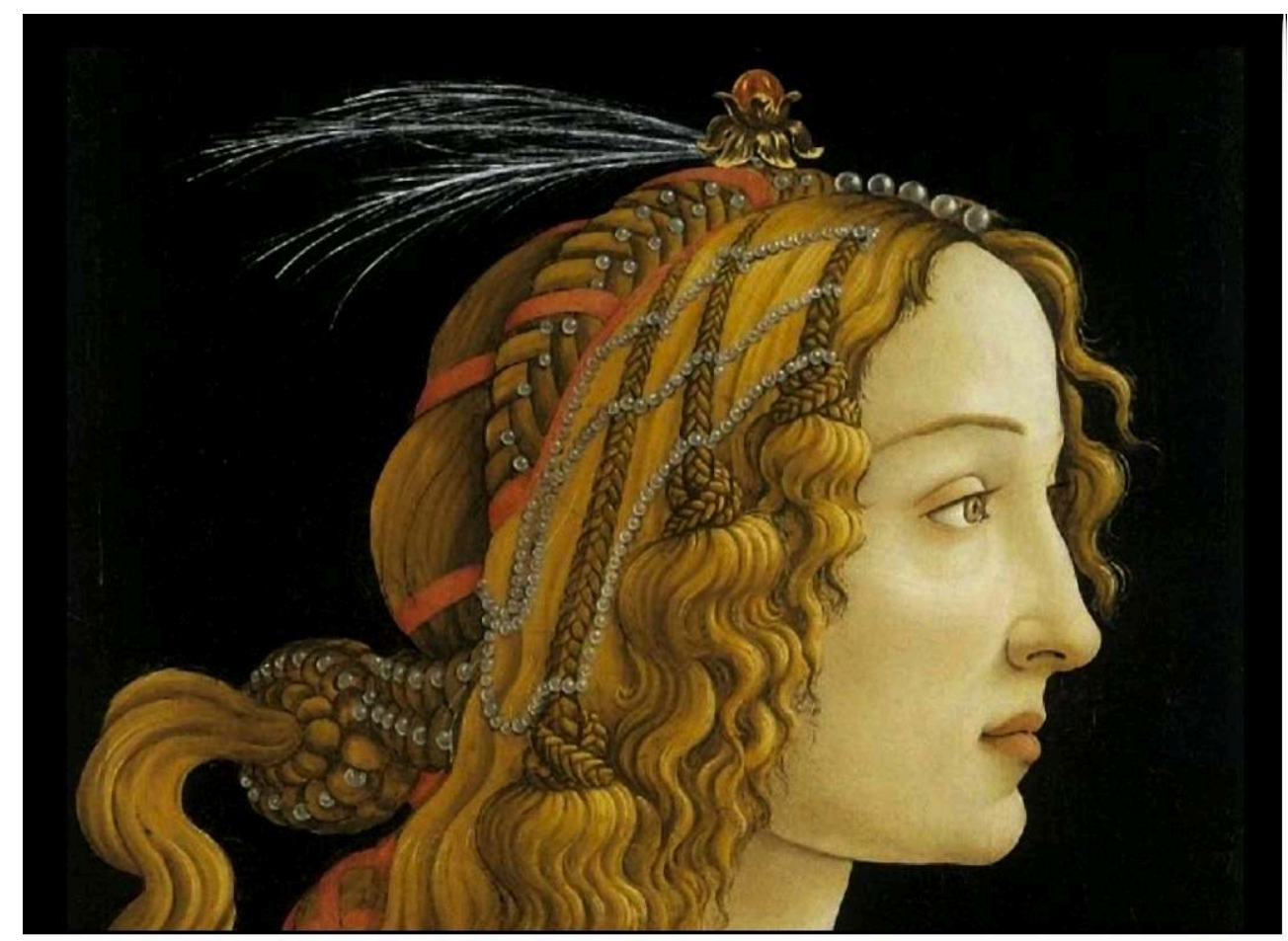

Atelier de S. Botticelli, Simonetta Vespucci (détail), Chantilly, Musée Condé

31 La parure sera donc d'autant plus mondaine qu'elle « ne ressemble à rien » : cheveux, poils, plumes, piquants... : c'est autant la forme organique humaine qui se désorganise que la forme animale elle-même. Les Indiens d'Amazonie, dans leur pratique extraordinaire de l'ars plumaria, ne laissent de désorganiser le corps humain autant que le corps de l'oiseau ${ }^{63}$. 
Fig. 20

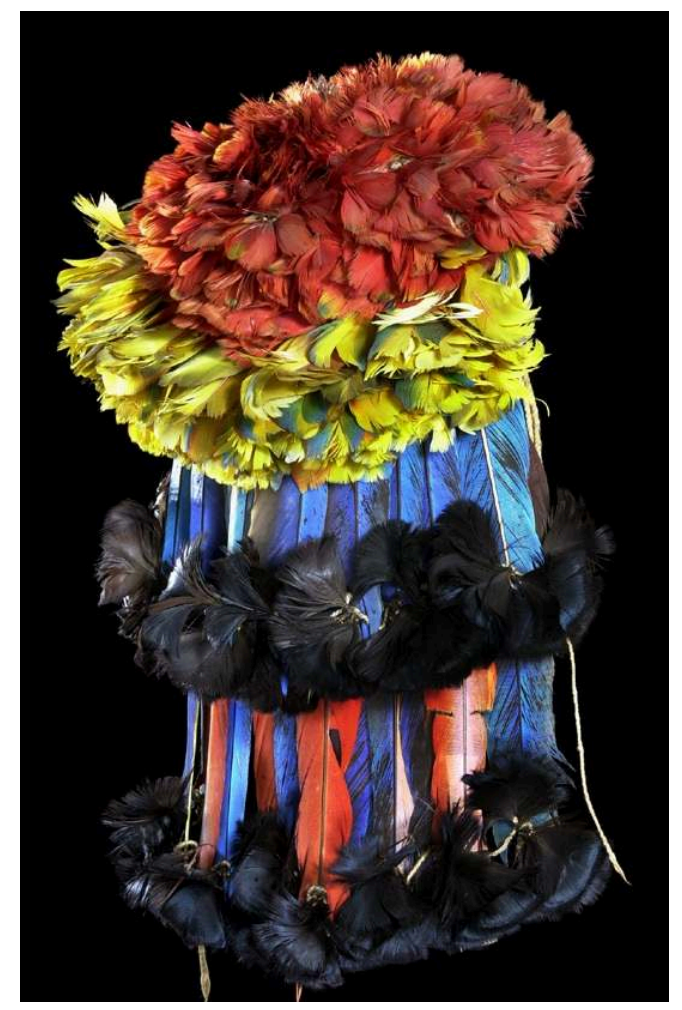

Coiffe couvre-nuque mundurucu, plumes et coton, Brésil, Rio Tapajos, Neuchâtel, Musée d'ethnographie

Fig. 21

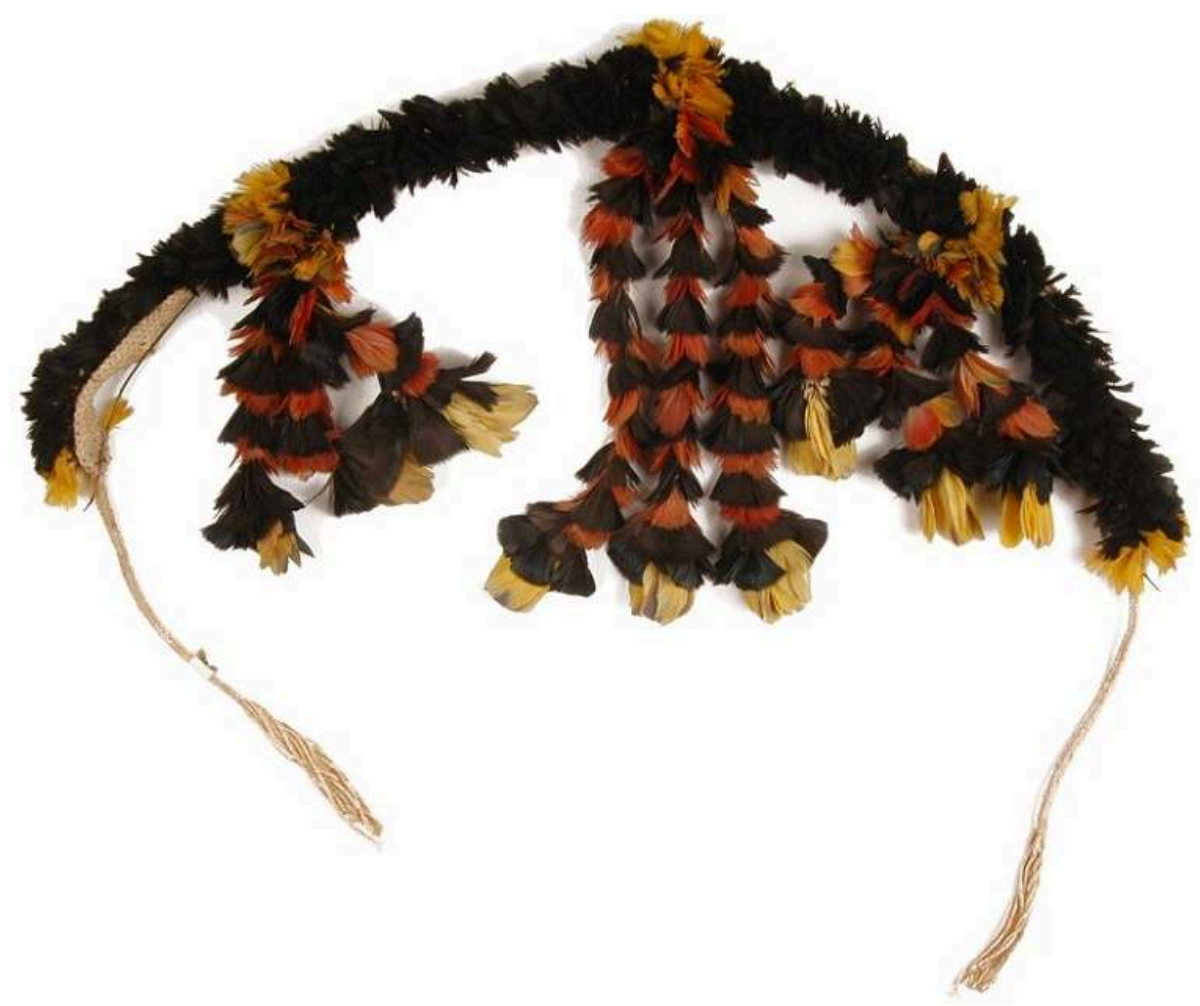

Ceinture mundurucu, plumes et coton, Brésil, Rio Tapajos, Paris, Musée du Quai Branly 
Ainsi, la plumasserie des Mundurucu de l'Amazonie centrale (fig. 20), qui portait ${ }^{64}$ peutêtre cet art à son niveau le plus achevé, se distingue-t-elle par la souveraine étrangeté de ses formes, qui n'a d'égale que sa suprême élégance ${ }^{65}$. La plume y perd notamment sa fonction organique de recouvrement (la plume décrivant essentiellement une forme longitudinale et superficielle) pour se transfigurer en une véritable construction conférant une improbable plasticité à la plume. Mais les formes corporelles humaines perdent autant en organicité que la matière organique qui les orne. La ceinture de la livrée de parade du guerrier mundurucu (fig. 21, 22) est ainsi remarquable pour ses pendants qui sur les cuisses et les fesses dessinent de véritables lignes abstraites qui contreviennent aussi bien à la forme radiale d'une ceinture qu'à l'unité des membres qui se trouvent ainsi désindividualisés.

Fig. 22

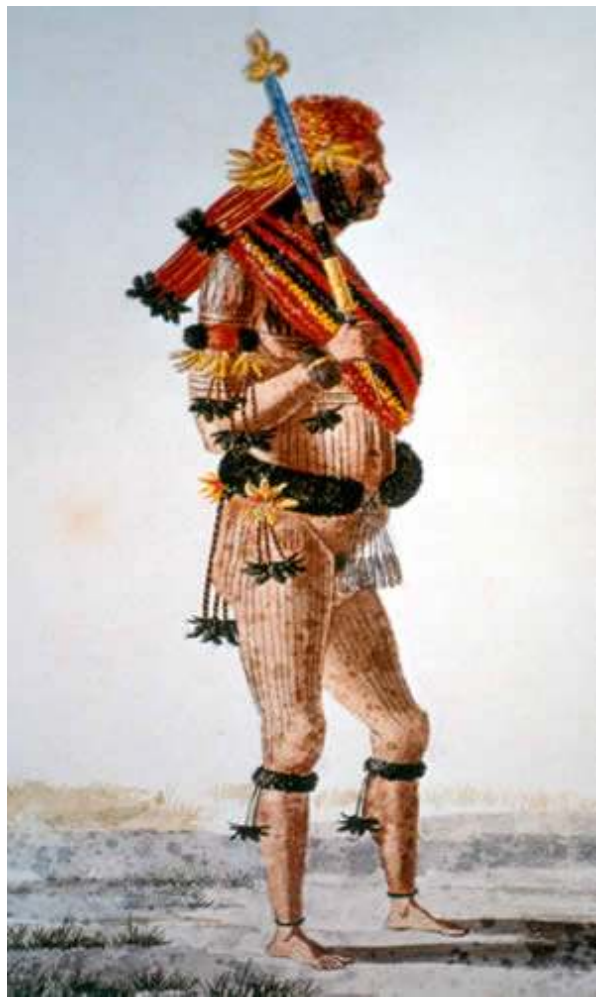

Hercule Florence, Guerrier Mundurucu, XIXe siècle, aquarelle

\section{Cosmétique animale}


Fig. 23

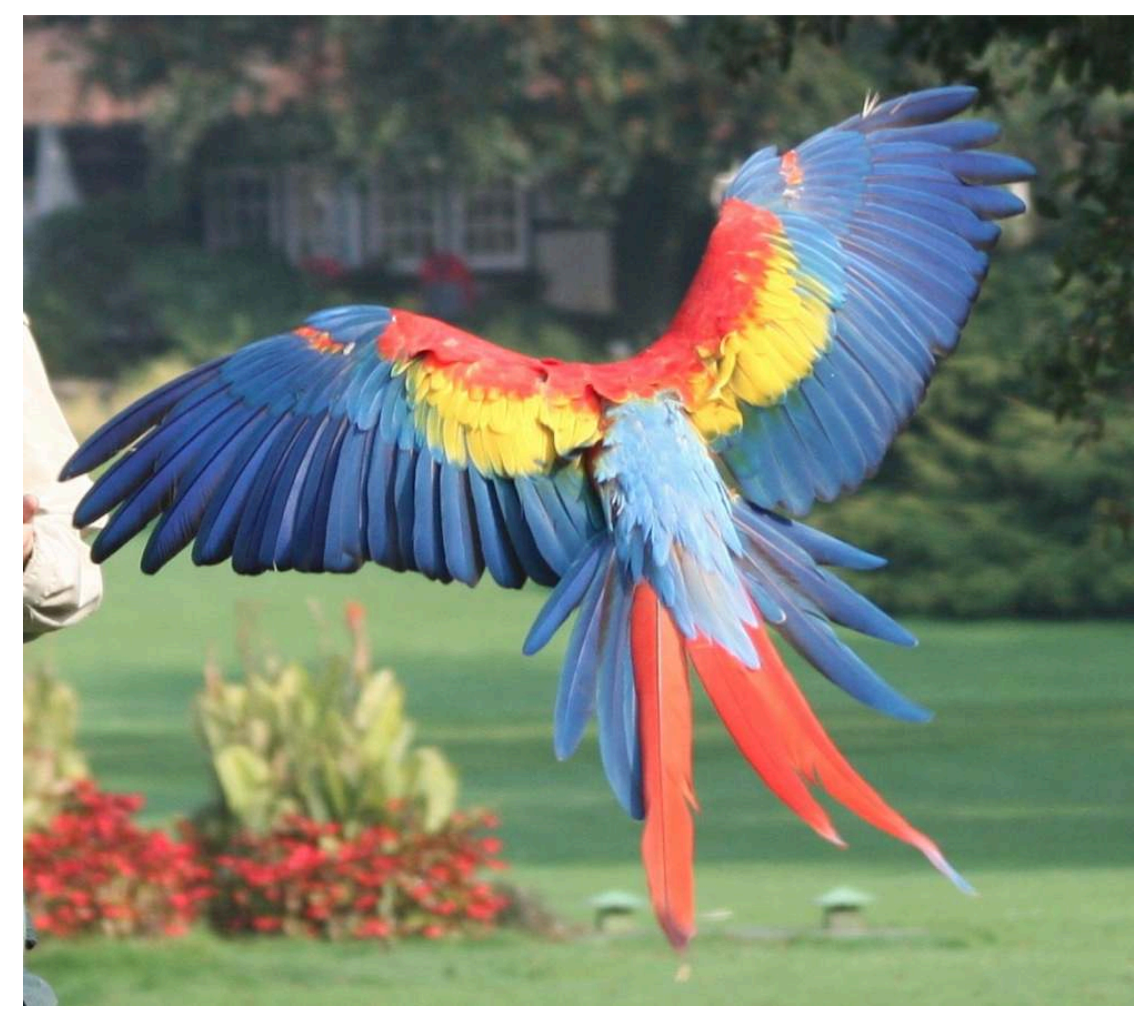

Ara Macao en vol

Mais plus que les hommes ce sont sans aucun doute les animaux qui sont passés maitres de l'anorganique. Zébrures, rayures, ocelles, taches, bandes, couleurs vives, irisées... : comment parler de tels phénomènes en termes de parure, autrement que par anthropomorphisme (fig. 23, 24) ? C'est que toutes ces formes et couleurs parcourent la surface comme autant de motifs autonomes qui ne peuvent se confondre avec le corps organique de l'animal. Quelque chose se décolle sans pour autant constituer un objet ou une forme substantielle. À sa façon, le grand zoologue Adolf Portmann a consacré tout son œuvre morphologique à rendre compte de cette étrange anorganicité animale, au titre d'une biologie de la parure animale ${ }^{66}$. Bien étrange "biologie» au demeurant, puisque le savant suisse ne visait pas tant des phénomènes formels et chromatiques que ce qui, dans ces phénomènes, les transfigure en d' " authentiques apparences " (eigentliche Erscheinungen), en d'autres termes, les rend expressifs. Il ne faudra pas s'étonner alors de passer subrepticement de l'organique au vital, du vivant à une vitalité plus profonde ${ }^{67}$. L'apparence expressive, de ce point de vue n'est ni organique, ni inorganique mais bien anorganique: les ocelles du jaguar autant que les couleurs chatoyantes des perroquets ne sont pas tout à fait inorganiques, puisque chimiquement, ce sont toujours des cellules vivantes qui les composent, puisque ce sont toujours des processus morphogénétiques qui président à leur formation; mais elles ne sont pourtant pas tout à fait organiques puisqu'elles ne se décalquent pas sur l'anatomie ou les divisions de l'organisme, et surtout, puisqu'elles demeurent le plus souvent a-fonctionnelles au regard de la conservation de l'espèce (voire s'y opposent, lorsque la parure devient encombrante au point de mettre en péril l'individu en l'handicapant dans ses mouvements ${ }^{68}$ ) ; la livrée des perroquets, pour garder le même exemple, ne connaît pas de dimorphisme sexuel et la vivacité des couleurs interdit 
toute idée de camouflage. Parler de parure animale est donc ici une façon de poser une question quant au sens de ces formes et non quant à leur formation ou leur fonction ${ }^{69}$.

Fig. 24

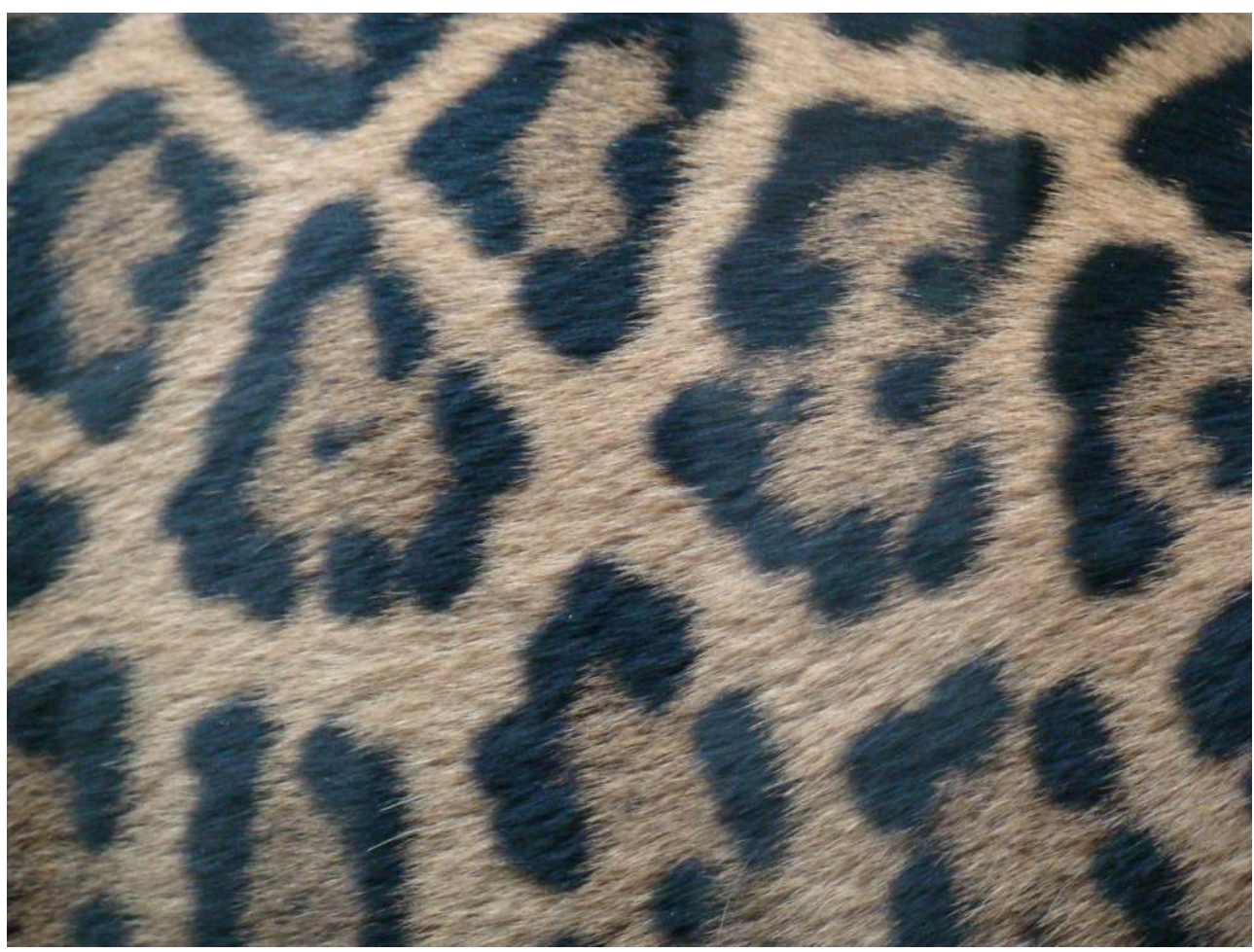

Ocelles de jaguar

Portmann n'aura de cesse de relever les régularités morphologiques par lesquelles les formes expressives, destinées à la vue se distinguent radicalement des formes inexpressives, non destinées à la vue (les organes internes par exemple), une distinction qui recoupe, à l'exception notable des animaux transparents ${ }^{70}$, une opposition extérieur-intérieur. Il y a parure animale dans la mesure où le motif n'est ni tout à fait intérieur, ni tout à fait extérieur : il ne se réduit pas à ses conditions internes de production, sans pour autant être projeté de l'extérieur. Dire que l'animal est strié, traversé, parcouru de lignes, motifs et couleurs, c'est encore une fois souligner l'extériorité incorporelle de ces formes, c'est insister sur ceci qu'une parure n'est pas plus projetée de l'extérieur (revêtement inorganique) qu'elle n'est produite de l'intérieur (formation organique) et qu'elle n'est donc pas réductible à un fait de culture, ni à un fait de nature : expression, elle devient avec le monde ${ }^{71}$.

À quelles conditions pourra-t-on alors parler d'une cosmétique animale? Il faut prendre garde ici à ne pas reconduire à notre insu l'interdiction que l'Occident antique, chrétien ou moderne aura posée quant à la possibilité de penser une parure animale. Autrement dit, il ne s'agit justement pas de prêter à l'animal les prérogatives techniques, esthétiques, fonctionnelles qui ont fait le titre d'humanité cosmétique. Il est ainsi remarquable de constater à quel point l'idée de vêtement animal ne peut se concevoir que sous un horizon anthropologique, sinon anthropocentrique :

L'homme ne peut pas être considéré comme le seul animal à se fabriquer ou à posséder des vêtements. Des chimpanzés du Sénégal se font des protections de pieds et de corps pour cueillir des fruits dans des épineux. Des bonobos sauvages 
prennent des feuilles en guise de parapluie, exactement comme on le faisait dans une région de l'Inde. Un crabe du détroit de Lembeh se "couvre" durablement de morceaux de feuilles à des fins de camouflage. Le bernard-l'ermite se donne une coquille de gastéropode et il en change plusieurs fois au cours de sa vie. Il est difficile de ne pas considérer cela comme des vêtements, dans la mesure où l'historiographie place la fonction protectrice en première ou deuxième place, à côté des fonctions de parure, de pudeur et de communication. On trouverait bien d'autres exemples de vêtements animaux qui respectent les définitions du vêtement humain, et aucune définition du vêtement ne parviendrait à exclure les cas animaux, en restant valable pour l'homme en général ${ }^{72}$.

Protection, couverture, abri: c'est bien le vêtement humain qui prête ici son dynamisme projectif au vêtement animal. Car la parure ou le vêtement est toujours pensé comme projection, dans les deux sens du terme : comme la production planifiée d'un artefact, autant que comme l'application de cet artefact, fût-il ready made, sur la surface du corps.

Fig. 25

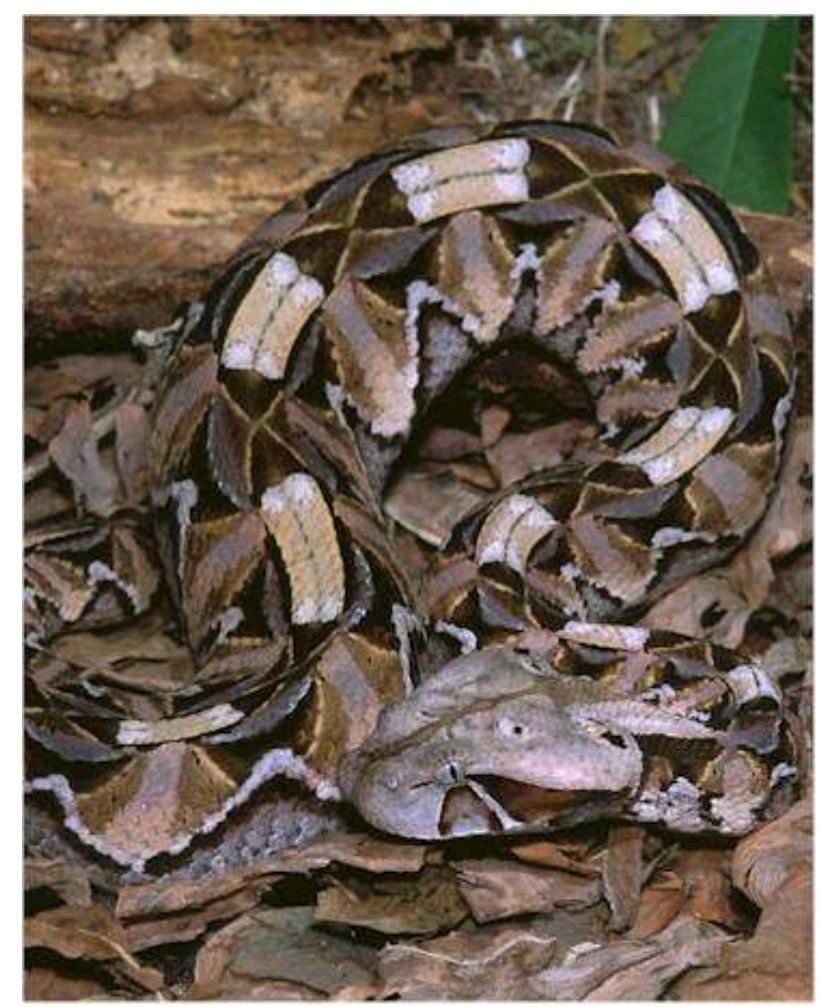

Camouflage disruptif : vipère du Gabon

Invoquer des fonctions naturelles de camouflage ne change pas grand-chose puisque l'on vise moins une utilité fonctionnelle qu'une efficacité esthétique. A supposer que l'on en tienne compte, en sachant que le camouflage ne saurait «expliquer » qu'une infime partie de la richesse expressive des parures animales, on ne pourra qu'y voir au maximum le vecteur d'un devenir-monde, au minimum une façon pour l'animal de composer esthétiquement et morphologiquement avec son milieu: devenirimperceptible. En sorte que c'est une même abstraction dépersonnalisante qui œuvre dans la parure humaine comme dans la parure animale. On dira de la parure ce qu'on dit d'un certain type de camouflage : qu'elle est disruptive : elle dilue l'individualité d'un 
corps en noyant ou en brisant ses contours ${ }^{73}$. Ainsi des motifs réticulés de très nombreux serpents, qui leur font perdre leur forme allongée caractéristique (fig. 25) ; ainsi des zèbres qui, amassés en troupeau, perdent leur individualité formelle (fig. 26). Pour ne rien dire évidemment des innombrables colorations et formes improbables qui transforment le lézard en écorce, le poisson en rocher, l'insecte en brin d'herbe, la mante en feuillage...

Fig. 26

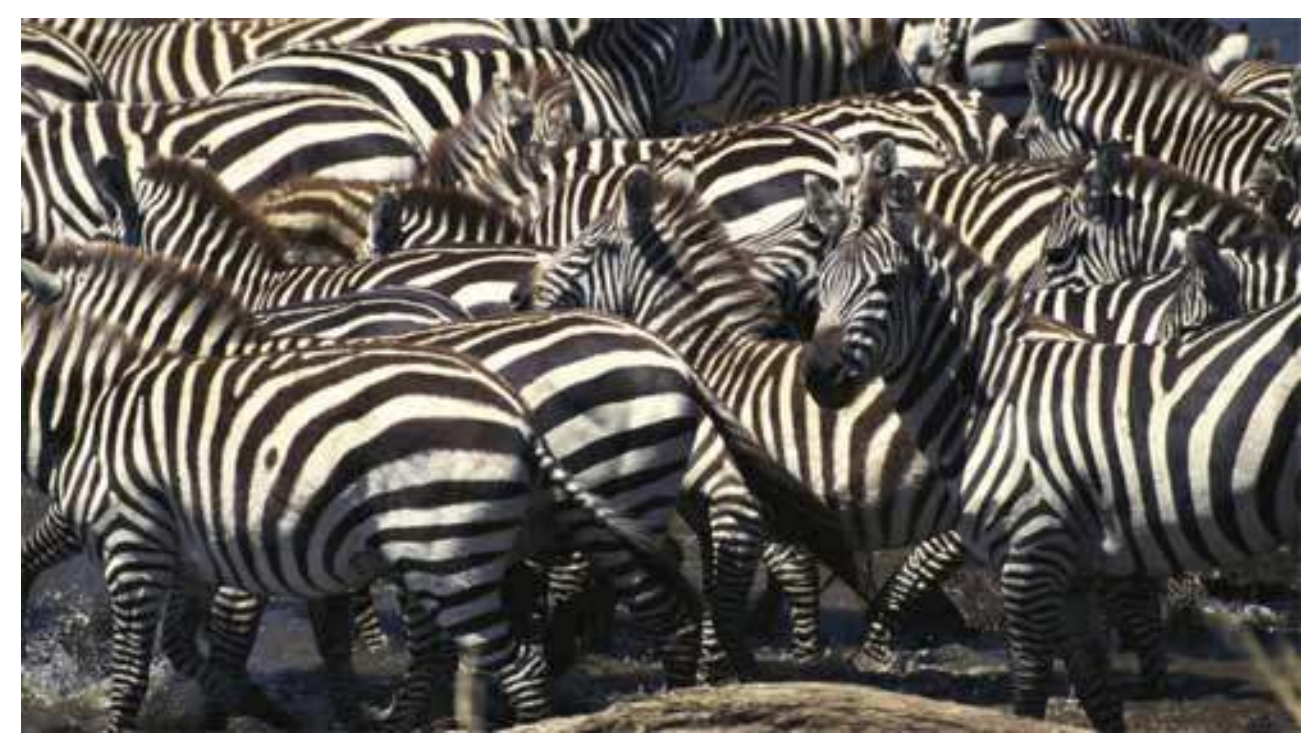

Camouflage disruptif : troupeau de zèbres

38 À la lettre, il n'y a pas camouflage, encore moins mimétisme ${ }^{74}$, mais une souveraine expression. Humaine ou animale, la parure implique le monde : elle le plie dans un motif, une couleur, un dessin qui traverse les stratifications naturelles (matière, vie, esprit). En sorte qu'une parure est toujours en même temps minérale et organique, symbolique et végétale, humaine et animale... Notre propre langage vestimentaire, avec ses boas, ses queues de pie, ses guêpières et autres aigrettes (et pour ne rien dire de l'ambivalence témoigne parfaitement de cette souveraine implication : ce ne sont là assurément pas des métaphores mais le germe de devenir - animal, fleur - qui filent droit vers un devenir-monde. Avec la parure, c'est le monde qui, transversalement, s'involue : l'unité cosmique ne se saisit pas comme Tout, mais s'éprouve en suivant des lignes ornementales chaosmiques, des traits expressifs. Les apparences ont ainsi regagné la Nature, si tant est que «nature » ne désigne ici pas autre chose que l'en soi de cette transversalité, qu'elle ne dessine pas autre chose que le plan de ces participations humaines, minérales, animales, végétales, et aucunement un socle commun et fondateur: "les participations, les noces contre nature, sont la vraie Nature qui traverse les règnes $»^{75}$. C'est ainsi qu'une cosmologie de la parure se fond dans une plus vaste histoire naturelle des apparences. 


\section{NOTES}

1. Homère, L'Illiade, XIV, 187.

2. M. Costantini, "Kosmos au "siècle" de Périclès ", in Histoires d'ornement, éd. P. Ceccarini, J.-L. Charvet, F. Cousinié, C. Leribault, Paris, Rome, Klincksieck, Académie de France à Rome - Villa Médicis, 2000, p. 45.

3. Tertullien, La Toilette des femmes, I, 4, 1-2, trad. M. Turcan, Paris, Cerf, 1971, p. 62-63. Sur la fondation cosmique de l'esthétique grecque, voir encore la présentation synthétique de G. Lombardo, L'Esthétique antique, Paris, Klincksieck, 2011, p. 13-24.

4. P.-A. Michaud, « Fashion victims. Mode et martyre selon Tertullien (vers 160-après 220), in id., Le Peuple des images. Essai d'anthropologie figurative, Paris, Desclée de Brouwer, 2002, p. 223.

5. Tertullien, La Toilette des femmes, II, 12, 3, op. cit., p. 156-157.

6. Sur la nudité et le vêtement chrétiens, voir notamment G. Bartholeyns, «L'homme au risque du vêtement. Un indice d'humanité dans la culture occidentale», in Adam et l'astragale. Essais d'anthropologie et d'histoire sur les limites de l'humain, éd. G. Bartholeyns, P. O. Dittmar, T. Golsenne, M. Har-Peled, V. Jolivet, Paris, Ed. de la MSH, 2009, 105-113.

7. Pour parler comme Jean-Claude Bonne. L'ornemental entretiendrait avec l'ornement le même rapport que le figural avec le figuratif.

8. J. Soulillou, Le livre de l'ornement et de la guerre, Marseille, Parenthèses, 2003, p. 96-97.

9. E. Benvéniste, Le vocabulaire des institutions indo-européennes. 2. Pouvoir, droit, religion, Paris, Minuit, 1969, p. 100.

10. Ibid., p. 101.

11. J.-C. Bonne, " De l'ornemental dans l'art médiéval (VIIe-XIIe siècle). Le modèle insulaire ", in J. Baschet et J. - C. Schmitt, L'image. Fonctions et usages des images dans l'occident médiéval, Paris, Cahiers du Léopard d'or 5, 1995, p. 237.

12. Edgar de Bruyne a largement insisté sur ce sens générique de musica: "C'est sur les proportions que se fonde la vision esthétique du monde. Les anciens eux-mêmes l'ont appelée musicale. Mais il est évident que ce terme ici n'a pas du tout la signification moderne : il est simplement synonyme d'esthétique et désigne toute harmonie quelle qu'elle soit ", cf. E. de Bruyne, Etudes d'esthétique médiévale (1946), Paris, Albin Michel, 1998, t. I, p. 11. Sur l'esthétique musicale, voir I, p. 306-338, I, 478-502, II, 227-238 et passim et sur l'harmonie universelle selon Boèce, cf. I, p. 9-26.

13. J. C. Bonne, « De l'ornemental dans l'art médiéval », art. cit., p. 238.

14. G. Semper, «De la détermination formelle de l'ornement et de sa signification comme symbole de l'art » (1856), trad. J. Soulillou in G. Semper. Du style et de l'architecture. Ecrits, 1834-1869, éd et trad. J. Soulillou, Marseille, Parenthèses, 2007, p. 235.

15. Cité par J. Soulilou, « Introduction », in. G. Semper, Du style et de l'architecture, op. cit., p. 27.

16. G. Semper, « De la détermination formelle de l'ornement », ibid., p. 236.

17. Ibid., p. 240.

18. Ibid., p. 243.

19. Ibid., p. 247.

20. Id., Der Stil in den technischen und tektonischen Künsten oder praktische Ästhetik : ein Handbuch für Techniker, Künstler und Kunstfreunde, Munich, 1860, trad. fr. partielle J. Soulillou, in G. Semper, Du style et de l'architecture, op. cit., p. 265-338.

21. G. Semper, Der stil (« Prolegomene »), trad. J. Soulillou, in G. Semper, op. cit., p. 291.

22. Ibid., p. 294.

23. Ibid., p. 295.

24. J. Soulillou, «Introduction », in G. Semper, op. cit., p. 28 
25. La conférence de 1856 se plaçait dès ses premières lignes sous l'invocation du cosmos grec. Cf. G. Semper, « De la détermination formelle de l'ornement...», op. cit., p. 235.

26. E. Gombrich, The Sense of Order. A study in the Psychology of decorative Art, Londres, Phaidon, 1979. L'aversion de Gombrich pour Semper, dans les quelques pages qu'il lui consacre («Semper is pedantic and soporific », p. 47), n'en devient que plus surprenante.

27. Ibid., p. 6.

28. Gombrich parlant p. 5 des « laws of physics ».

29. Ibid., p. 1.

30. Kant, Critique de la raison pure (1787), trad. A. Tremesaygues et B. Pacaud, Paris, PUF, 1993 p. 142: «L'entendement n'est donc pas simplement un pouvoir de se faire des règles par la comparaison des phénomènes, il est lui-même une législation pour la nature, c'est-à-dire que sans entendement, il n'y aurait nulle part de nature, je veux dire d'unité synthétique du divers des phénomènes d'après des règles ".

31. E. Gombrich, The Sense of Order, op. cit., p. 117.

32. Pour reprendre le titre du dernier chapitre, «The Edge of Chaos ».

33. Charles Blanc, L'art dans la parure et le vêtement, Paris, Librairie Renouard, 1875, p. $4:$ « ... les motifs sans nombre que les hommes ont inventés jusqu'à ce jour et ceux qu'ils inventeront encore pour orner leurs personnes, leurs demeures ou leurs temples, sont engendrés par l'application de l'un de ces cinq principes que nous allons énoncer : la répétition, l'alternance, la symétrie, la progression, la confusion. Telles sont les sources auxquelles on peut ramener tous les ornements dont l'idée a été empruntée de la nature, et que l'homme a soumis aux lois de son esprit et à l'empire de sa liberté ». et p. 41 : «mais chacun de ces principes est accompagné d'un élément secondaire qui en dérive et qui, venant multiplier encore les ressources du décorateur, lui permet de varier ses combinaisons à l'infini. (Ainsi) à la répétition se rattache la consonance ; à l'alternance, le contraste ; à la symétrie le rayonnement; à la progression, la gradation; à la confusion pondérée, la complication réfléchie ».

34. Voir Gombrich, op. cit., p. 70-72. On sait le sort qu'un artiste comme Sol LeWitt aura réservé à l'épuisement méthodique du cube. Voir à ce sujet la belle étude de R. Krauss, «LeWitt in progress ", in L'Originalité de l'avant-garde et autres mythes modernistes, trad. J.-P. Criqui, Paris, Macula, 1993, p. 335-350.

35. Sur cette extraordinaire parure, sans doute la plus grande que l'on trouve en Amazonie, voir

G. Verswijver, Kaiapo. Materielle Kultur-spirituelle Welt. Material culture-spiritual world, Museum für Völkerkunde, Francfort sur le Main, 1995, p. 114-124.

36. Ce que remarquait justement Ernst Gombrich : "in adorning the body an order is surimposed on an existing order, respecting or sometimes contradicting the symmetries of the organic form ", The Sense of Order, op. cit., p. 65.

37. Voir l'ouvrage classique d'Alexandre Koyré, Du Monde clos à l'univers infini, Paris, Gallimard, 1973.

38. G. Deleuze, Proust et les signes, Paris, PUF, 1964, p. 193-194.

39. Pour parler comme Pierre Montebello, dans son livre L'Autre métaphysique. Essai sur Ravaisson, Tarde, Nietzsche et Bergson, Paris, Desclée de Brouwer, 2003.

40. Pour une présentation critique et bibliographique de l'actualité de la question, voir G. Bartholeyns, «Faire de l'anthropologie esthétique », Introduction au dossier « Les apparences de l'homme ", Civilisations, vol. 59, n², 2011, p. 9-40.

41. M. Mauss, Manuel d'ethnographie, Paris, Payot, 1967, p. 96.

42. Ibid., p. 96-99.

43. Ibid., p. 99-102.

44. Ibid., p. 96-97 : « (la cosmétique) comprend la beauté surajoutée au corps [...]». 
45. Pour ne rien dire des très nombreuses déformations corporelles produites par le vêtement lui-même : des pieds bandés des Chinoises au corset occidental. Voir là-dessus Odile Blanc, Vivre habillé, Paris, Klincksieck, 2009, p. 67-68.

46. M. Mauss, Manuel d'ethnographie, op. cit., p. 96 : «L'homme a toujours cherché à se surajouter quelque chose de beau en société, à se l'incorporer ".

47. Voir A. Warburg, Souvenir d'un voyage en pays Pueblo. Notes inédites pour la conférence de Kreuzlingen sur le rituel du serpent (1923), trad. S. Muller, in P.-A. Michaud, Aby Warburg et l'image en mouvement, Paris, Macula, 1998, p. 264-265 : «Tragédie de l'incorporation phénoménologique. Une extension tragique parce qu'elle ne correspondait pas à son être. [...] D'où viennent toutes ces questions et ces énigmes sur l'empathie devant la nature inanimée ? Parce qu'il existe effectivement pour l'homme un état qui peut l'unir à quelque chose - justement en portant ou en maniant quelque chose - avec quelque chose qui lui correspond, mais qui ne coule pas dans ses veines. Le tragique du costume et de l'outil est l'histoire de la tragédie humaine [...] ».

48. Gil Bartholeyns a bien analysé ce propre vestimentaire de l'homme en occident; voir "L'homme au risque du vêtement ", art. cit.

49. Voir G. Semper, Der stil, op. cit. Il ne nous revient pas ici de s'étendre plus longuement sur la fiction sempérienne et sa fécondité théorique.

50. J. Soulillou, Le livre de l'ornement et de la guerre, op. cit., p. 108.

51. Ibid., p. 111. Ainsi de Viollet le Duc qui dénonce l'architecte romaine au titre de ce qu'elle " n'est qu'un vêtement qui n'est pas toujours parfaitement adapté à la chose qu'il recouvre » cité ibid., p. 111.

52. Voir O. Blanc, Vivre habillé, op. cit., p. 68: "L'histoire du vêtement est celle des transformations qu'il fait subir au corps naturel : une orthopédie des apparences ».

53. Odile Blanc va même plus loin en sexualisant le corps du vêtement. Cf. ibid., p. 70 (« Le vêtement a-t-il un corps?»), l'auteur jouant par ailleurs sur le sens du « corps », cet « accessoire apparu à la fin du XVe siècle, et porté sur la chemise qui étrangle la taille et efface le ventre » (p. 67).

54. J. Soulillou, Le livre de l'ornement et de la guerre, op. cit., p. 108.

55. G. Simmel, « Psychologie de la parure ", in La parure et autres essais, Paris, éditions de la MSH, 1998, p. 82-83.

56. Sur les incorporels stoïciens, voir la toujours pertinente étude d'E. Bréhier, La Théorie des incorporels dans l'ancien stoïcisme, Paris, Vrin, 1928. On sait par ailleurs le sort que Gilles Deleuze a réservé à ces incorporels, notamment dans sa théorie de l'événement dans Logique du sens, Paris, Minuit, 1969.

57. Voir J.-C. Martin, Parures d'Eros. Un traité du superficiel, Paris, Kimé, 2003.

58. Ibid., p. 129.

59. E. Coccia, La Vie sensible, trad. M. Rueff, Paris, Rivages, 2010, p. 124 (nous soulignons).

60. G. Deleuze et F. Guattari, Capitalisme et schizophrénie 2. Mille plateaux, Paris, Minuit, 1980, p. 343. Sur le concept deleuzien de monde, voir notamment les analyses d'A. Beaulieu, Gilles Deleuze et la phénoménologie, Mons, Sils Maria, 2004, p. 17-74.

61. G. Deleuze et F. Guattari, Mille plateaux, op. cit., p. 342.

62. A proprement parler, le postiche sert à pallier une calvitie partielle voire à modifier localement la forme de la coiffure, mais pas à moduler en totalité son aspect.

63. Pour de plus amples développements, nous nous permettons de renvoyer à notre étude, "L'ars plumaria en Amazonie. Pour une esthétique mineure », Civilisations, vol. 59, n², 2011, p. 87-108.

64. Les Mundurucu (groupe de langue Tupi) ont perdu toute leur plumasserie depuis près d'un siècle.

65. Sur la plumasserie mundurucu, voir D. Campana, «L'arte plumaria dei Mundurucu (Brasile) e di altri popoli del Sud-America ", Archivio per l'antropologia e la etnologia, n³5, 1905, p. 177-197. 
66. Voir A. Portmann, La forme animale, trad. G. Remy, Paris, Payot, 1961. Pour une présentation de l'œuvre portmannienne et de ses enjeux, voir dans cette même revue B. Prévost, «L'élégance animale. Esthétique et zoologie selon Adolf Portmann », Images Re-vues, 6 (Devenir-animal), 2009 (http://imagesrevues.revues.org/379).

67. « Paraître est une fonction vitale » écrit Portmann (Préface à T. Jahn, La vie et ses formes, Paris, Bordas, 1968, p. 13) : il est très important que Portmann écrive « vitale » et non « organique ».

68. Ce point est capital et nécessiterait un long développement puisqu'il témoigne non plus seulement d'une divergence mais bien d'une opposition entre la vie organique de l'individu et la vitalité de l'apparence.

69. Voir A. Portmann, "Was bedeutet uns die lebendige Gestalt?", Neue Sammlung, Göttinger Blatter für Kultur und Erziehung, 6 Jahrgang, Heft 1, Janv. Fév. 1966, p 1-7 (le thème du sens des apparences, opposé à celui de la genèse des formes parcourt toute l'œuvre de Portmann).

70. Puisque leur transparence rend justement visibles leurs organes internes qui se voient ainsi dotés des prérogatives des organes de l'apparence (coloration vive, symétrie, individuation formelle, etc.).

71. Thomas Golsenne a récemment développé plusieurs thèses sur la parure sensiblement voisines des nôtres. Voir T. Golsenne, «Généalogie de la parure. Du blason comme modèle sémiotique au tissu comme modèle organique ", Civilisations, vol. 59, $\mathrm{n}^{\circ} 2,2011$ ("Les apparences de l'homme »), p. 41-58. Nous le retrouvons en effet sur des points essentiels parmi lesquels la critique du privilège anthropologique de la parure et la séparation nature/culture qu'elle induit, la remise en cause du modèle projectif, la référence à l'œuvre de Semper, de Portmann, etc. Cependant, la proximité semble davantage tenir aux déclarations d'intention théorique qu'à leur mise en œuvre critique puisque l'auteur reste attaché à un profond naturalisme qui contrevient manifestement à la volonté affichée de «mise en cause du modèle naturaliste » au profit d'une « conception plus vitaliste de la parure ». La morphogenèse et ses modèles physico-chimiques est en effet convoquée pour penser la naturalité de la parure, en vertu de processus tous peu ou prou liés à la croissance. Mais si la distinction nature/culture est ainsi temporairement neutralisée, elle revient au plus vite dans la distinction entre deux dynamismes de parure: projection inorganique depuis l'extérieur d'une part, «poussée vitale de l'intérieur » de l'autre. " Nature » est ainsi le nom de cette intériorité qui agit dans les choses, inertes ou vivantes, au titre de "forces physiques universelles ». C'est là retrouver le geste fondateur de tout naturalisme et de sa conception mécaniste et explicative de la force, qui ne fait qu'intérioriser des chocs extérieurs («la parure animale [...] est apparue par le résultat de l'effet de forces physiques sur les matériaux du vivant »).

72. G. Bartholeyns, «L'homme au risque du vêtement », art. cit., p. 125-126.

73. Voir R. Caillois, Le mimétisme animal, Paris, Hachette, 1963, p. 25.

74. Rappelons que le camouflage désigne plutôt les relations de l'individu au milieu, tandis que le mimétisme concerne les rapports d'imitation entre espèce.

75. G. Deleuze et F. Guattari, Mille plateaux, op. cit., p. 295. Nous avons pour notre compte commencé à repenser le statut des apparences animales via la notion portmanienne d' "apparence sans destinataire», voir B. Prévost, "Les apparences inadressées. Usages de Portmann (doutes sur le spectateur) ", in L'adresse. XVIe colloque du Cicada, éd. B. Prévost et B. Rougé, Pau, Presses Universitaires de Pau (sous presse) (pré-publication internet: http:// www.fabula.org/atelier.php?Les_apparences_inadress\%26eacute\%3Bes). 


\section{RÉSUMÉS}

On interroge ici la dimension cosmique de la parure : qu'y a-t-il de mondain dans une coiffe, un maquillage voire un ornement guerrier? En quoi le pli d'une robe s'accorde-t-il avec le monde? Il ne faut pas se méprendre sur la tonalité grecque de cette question. Les Grecs Anciens ont certes formalisé l'articulation du cosmique et du cosmétique, mais cette articulation outrepasse largement ce cadre pour être encore à l'œuvre dans l'esthétique ornementale chrétienne médiévale, dans l'œuvre de Gottfried Semper ou même jusque dans le grand livre de Gombrich sur l'ornement. Plus précisément, on tente de se placer au-delà du principe qui préside toujours à l'ajointement cosmique-cosmétique, à savoir : une analogie d'ordre. Tout l'enjeu est alors de poser les conditions d'un devenir-monde de la parure réel et continu. La question de l'adéquation entre corps et parure, ou plutôt de son inadéquation, sert notamment de cheville critique pour penser une impersonnalité de la parure par laquelle le corps se décorpore et devient « anorganiquement » avec le monde.

We interrogate here the cosmic dimension of body ornament : in which sense a headdress, a make up or a warrior adornement can be said "wordly " ? How the fold of a dress fits with the world? We must not misunderstand the greek tone of the question. The Ancient Greeks have certainly formalized the articulation of cosmic and cosmetics, but this joint goes beyond this framework to be largely still at work in the ornamental medieval christian aesthetics, in Gottfried Semper's work, or even into Gombrich's great book on ornament. Specifically, we try to override the principle that always governs the ajointement cosmic-cosmetics, namely: an analogy of order. The very problem is then to set up the conditions of a real and continuous becoming-world of adornment. The question of the adequacy of body adornment, or rather its inadequacy, is particularly critical to think of an impersonal ornament, by which the body loses his bodyness and becomes "anorganically" with the world.

\section{INDEX}

Thèmes : anthropologie de l'art, esthétique, théorie de l'art

Keywords : Ornament, adornment, aesthetics, ethnology, ethology, cosmetics, Gottfried Semper, Georg Simmel, Adolf Portmann, Amazonia, camouflage

Mots-clés : Ornement, parure, esthétique, ethnologie, éthologie, cosmétique, Gottfried Semper, Georg Simmel, Adolf Portmann, Amazonie, camouflage

\section{AUTEUR}

\section{BERTRAND PRÉVOST}

Bertrand Prévost, philosophe et historien de l'art, est maître de conférences à l'Université Michel de Montaigne - Bordeaux 3. Il a notamment publié La Peinture en actes. Gestes et manières dans l'Italie de la Renaissance, Arles, Actes Sud, 2007, Botticelli. Le manège allégorique, Paris, éd. 1 :1, 2011, L'humaniste, le peintre et le philosophe. Théorie de l'art selon Leon Battista Alberti (sous presse). Il consacre l'essentiel de ses recherches à l'idée d'une cosmétique élargie. 